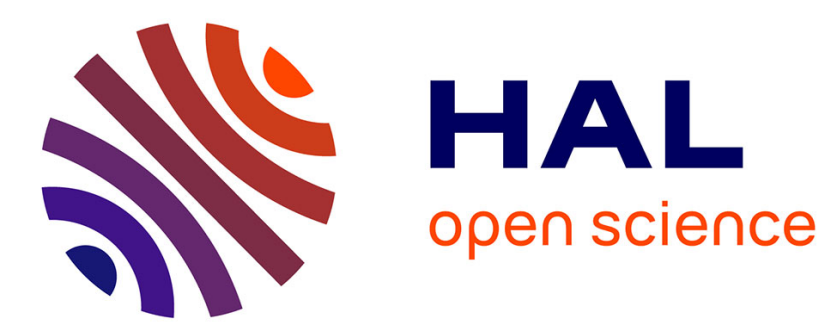

\title{
Well balanced residual distribution for the ALE spherical shallow water equations on moving adaptive meshes
}

\author{
Luca Arpaia, Mario Ricchiuto
}

\section{To cite this version:}

Luca Arpaia, Mario Ricchiuto. Well balanced residual distribution for the ALE spherical shallow water equations on moving adaptive meshes. Journal of Computational Physics, 2020, 405, pp.109173. 10.1016/j.jcp.2019.109173 . hal-02422335

\section{HAL Id: hal-02422335 \\ https://hal.inria.fr/hal-02422335}

Submitted on 21 Dec 2019

HAL is a multi-disciplinary open access archive for the deposit and dissemination of scientific research documents, whether they are published or not. The documents may come from teaching and research institutions in France or abroad, or from public or private research centers.
L'archive ouverte pluridisciplinaire HAL, est destinée au dépôt et à la diffusion de documents scientifiques de niveau recherche, publiés ou non, émanant des établissements d'enseignement et de recherche français ou étrangers, des laboratoires publics ou privés. 


\title{
Well balanced residual distribution for the ALE spherical shallow water equations on moving adaptive meshes
}

\author{
Luca Arpaia and Mario Ricchiuto \\ Coastal Risk and Climate Change Unit, French Geological Survey \\ 3 Av. C. Guillermin 45060 Orléans Cedex 2, France \\ and \\ Team CARDAMOM, Inria Bordeaux Sud-Ouest, \\ 200 av. de la vieille tour, 33405 Talence cedex, France
}

\begin{abstract}
We consider the numerical approximation of the Shallow Water Equations (SWEs) in spherical geometry for oceanographic applications. To provide enhanced resolution of moving fronts present in the flow we consider adaptive discrete approximations on moving triangulations of the sphere. To this end, we restate all Arbitrary Lagrangian Eulerian (ALE) transport formulas, as well as the volume transformation laws, for a 2D manifold. Using these results, we write the set of ALE-SWEs on the sphere. We then propose a Residual Distribution discrete approximation of the governing equations. Classical properties as the DGCL and the C-property (well balancedness) are reformulated in this more general context. An adaptive mesh movement strategy is proposed. The discrete framework obtained is thoroughly tested on standard benchmarks in large scale oceanography to prove their potential as well as the advantage brought by the adaptive mesh movement.
\end{abstract}

Keywords: Shallow Water equations; curvilinear coordinates; Arbitrary Lagrangian Eulerian formulation; Moving Mesh ; Residual Distribution; Well-Balanced

\section{Introduction}

The Earth's curvature has a strong impact on wave propagation at large scales. For this reason, efficient and accurate techniques to solve flow equations on the sphere are of interest in many fields, such as ocean modeling, weather prediction as well as coastal hazards. Our objective is to explore the possibility 5 of improving the discrete resolution of flows relevant to large-scale oceanographic applications by adap6 tively deforming an initial mesh, possibly already adapted to some of the data (e.g. initial depth or depth

Email address: 1.arpaia@brgm.fr, mario.ricchiuto@inria.fr (Luca Arpaia and Mario Ricchiuto) 
7 at rest). Indeed, mesh adaptation is useful to handle not only complex wave patterns, but also complex

8 bathymetries and coastlines. In this respect, the possibility of working on triangular grids may be useful

9 especially when approaching shallow areas. On these triangulations, in the spirit of the work done in ArR: 17

10 [1, we aim to assess the potential of adaptive mesh deformation, as well as its limitations for complex

11 multi-scale computations.

12

To achieve this objective, there are several aspects to be dealt with. The first one is the discrete formulation of the Shallow Water Equations (SWEs) on the sphere. The literature on the topic is quite large. On structured quadrilateral grids, it is common to formulate the SWEs in covariant curvilinear coordinates (with the set of covariant coordinates identified by a patchwise parametrization of the sphere). In the finite volume context, a thorough analysis is discussed in [2, 2 , 04 , Ros: 06 and other formulations are proposed $\mathrm{Ul} \mathrm{J}: 10$, Cas: 18 e.g. in 4 , 5]. On triangular meshes most works focus on high order local finite element-like mappings as in e.g. $\frac{\text { Comb:09, Bern:09 }}{6}$, or on a fully 3D formulation with Lagrange multipliers to locally re-project vectors on the appropriate basis. A compromise is the initial work of $\frac{R}{8}, 1 \mathrm{~s}: 13$ which covariant coordinates are combined with triangular grids and with a Residual Distribution (RD) discretization. An ad-hoc patching at the poles guarantees the $C^{0}$ continuity of the solution. This provides us with an initial framework to extend our previous work. In particular, we have shown in $\frac{\mathrm{Ri} B \cdot 09, \mathrm{~g} 11, \mathrm{Ric}, 15}{11}$, that RD method allows to handle very naturally source terms with a simple design of schemes preserving many known steady equilibria on unstructured triangulations. This is relevant when considering the SWEs in curvilinear coordinates which embed several geometrical and physical forcing terms. The RD method also allows to combine high order with monotonicity and positivity preservation principles. The work of $\frac{R}{8}$ : 13 however is limited to steady state problems, on fixed grids, with constant bathymetry, and linear schemes. Here we present a more general development of nonlinear second order discrete approximations, for steady and time dependent problems on complex bathymetries.

Another important aspect is the design of the adaptation strategy on the sphere. The geophysics community has focused on quad-tree AMR techniques based on cell subdivision, see for example | 12 McU: 15.Fer: 16 LGB: 11 Pop: 11 or 13, 14, and 15, 16] for tsunami propagation. Here we consider a different approach, consisting in generating an initial grid adapted to the problem's data (e.g. topography) and then using mesh deformation to track more accurately the flow features. An elegant way to deal with the underlying moving mesh is to formulate balance laws directly in an Arbitrary Lagrangian Eulerian (ALE) moving reference

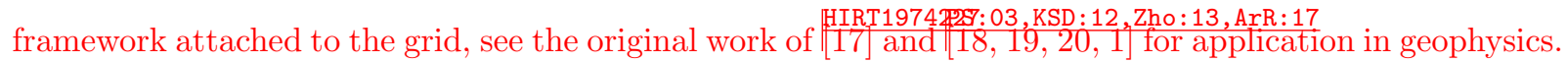


Given an ALE map $x=A(X, t)$ between a reference (fixed) domain and an actual (moving) one, there are two ways to formulate the PDEs in ALE form: either with respect to the reference coordinates $X$ or the actual ones $x$. In the former case, the derivation of the ALE equations in the reference domain becomes immediate in a spacetime setting: the transformation of the 4-divergence differential operator under the ALE map introduces an additional ALE transport term. An overview of the fundamental relations necessary to achieve this in a spacetime setting are discussed in e.g. 21 (or chapter 2 in

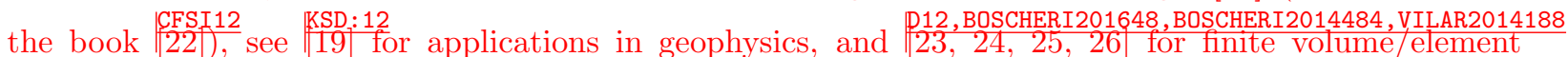
formulations on curved meshes based on similar principles. There is certainly a similarity in the use of the reference coordinates $X$ to represent the mesh movement and the aforementioned use of curvilinear coordinates to represent a geometrical manifold. Such analogy between the ALE form of balance laws and e.g. the governing equations of relativistic hydrodynamics, which are written in a time-dependent curved spacetime, is discussed in $\frac{t 16}{27 .}$.

In moving mesh applications, another strategy used very often is to express the differential operators in the actual configuration, and thus discretize them on the actual (moving) mesh. Within such approach, a special role is played by the Geometric Conservation Law (GCL), which enforces conservation of the

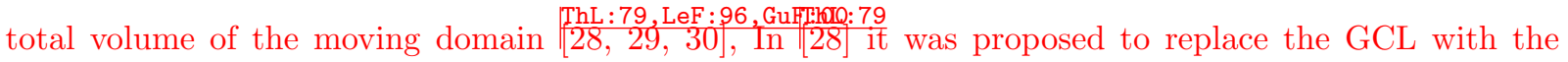
constraint of reproducing exactly uniform flows, and in $\left[\frac{\mathrm{GWF}: 00}{30}\right.$ it has been shown that satisfying the Geometric Conservation Law (GCL) is a sufficient condition for first order time accuracy on moving meshes. The compliance of the GCL at a discrete level is referred to as Discrete GCL (DGCL). Although rooted in fundamental relations of continuous mechanics $21,[22$, the practical importance/benefit of the GCL and of its discrete counterpart is sometimes controversial, see e.g. Fti: 131.

The approach that we use is of mixed type $\frac{\mathrm{SaA}: 08}{32} \cdot{ }^{2}$ The spherical map in written with respect to the curvilinear covariant coordinates. This means that the parametrization of the sphere is embedded in the

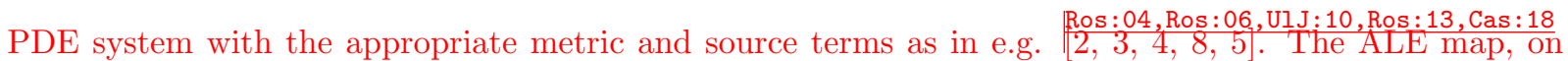
the contrary is written in the actual configuration, which does not require the computation of the ALE Jacobian matrix. In this setting, the ALE-SWEs on a 2D manifold will be derived using classical transport formulas and volume conservation statement. A derivation of the governing equations in spacetime is also given in the Appendix. It will be shown that, for the SWEs on the sphere, the satisfaction of a DGCL is a necessary condition to retain well-balancedness. To this end, a new ALE closure for RD schemes is proposed, since the one of $\frac{A r R}{33}: 14$ does not extend to the sphere, and more in general to manifolds.

The schemes obtained are thoroughly tested both on fixed and moving adaptive meshes on classical 
applications for the shallow water on the sphere, and on a realistic computation of relevance in oceanography: the regional propagation and coastal impact of the 2011 Tohoku-Honsu tsunami.

The paper is organized as follows. The standard SWEs in spherical geometry are recalled in section 2. Sections 3 and 14 are then devoted to the ALE-SWEs formulation on the sphere. The main notation and ingredients required for the discrete approximation are the introduced in section that These are used in section $\frac{\mid s e c: R D}{6}$ to construct well balanced second order residual distribution. The mesh movement strategy is

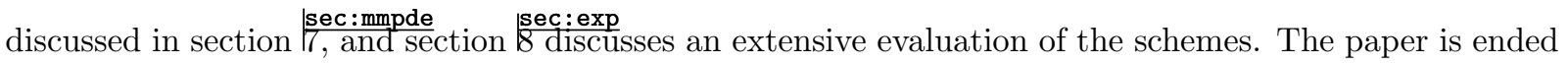
by a summary and an outlook on future developments.

\section{SWEs on the sphere}

In this section we recall the Shallow Water Equations (SWEs) on the sphere in covariant curvilinear coordinates. At every point of the sphere $\mathcal{S}^{2}$ we fix an orthogonal but not orthonormal reference system with covariant basis vectors $\left\{\boldsymbol{g}_{1}, \boldsymbol{g}_{2}\right\}$ together with local curvilinear coordinates $\left\{x^{1}, x^{2}\right\}$. The vectors $\boldsymbol{g}_{1}$ and $\boldsymbol{g}_{2}$ define the tangent plane to the sphere and are used to construct all quantities allowing to locally describe the manifold. For completeness, in $\frac{\mid a p p \cdot a 1}{A p p e n d i x}$ A.1 we recall some basic concepts from differential geometry, as well as many definitions used throughout the following sections. We will consider the evolution of the fluid depth $h(\boldsymbol{x}, t)$ measured along the radial axis. The bathymetry/topography height with respect to a given reference level is denoted by $b(\boldsymbol{x})$. The fluid free surface is defined here as $\eta=h+b$. We denote $h u^{i}$ the components of the discharge vector $h \boldsymbol{u}=h u^{i} \boldsymbol{g}_{i}$. We remark that the velocity components are not defined in a unitary basis. The SWEs in covariant curvilinear coordinates write 1

$$
\begin{gathered}
\frac{\partial h}{\partial t}+\frac{1}{\sqrt{G}} \frac{\partial}{\partial x^{j}}\left(\sqrt{G} h u^{j}\right)=0 \\
\frac{\partial h u^{i}}{\partial t}+\frac{1}{\sqrt{G}} \frac{\partial}{\partial x^{j}}\left(\sqrt{G} T^{i j}\right)+S^{i}=0
\end{gathered}
$$

$G$ denotes the determinant of the metric tensor defined in are the components of the momentum flux $\boldsymbol{T}=T^{i j} \boldsymbol{g}_{i} \boldsymbol{g}_{j}$, involving the components of the inverse metric tensor, also defined in the appendix.

The source term in the momentum balance reads

$$
S^{i}=G^{i j} g h \frac{\partial b}{\partial x^{j}}+c_{F} h u^{i}+S_{\gamma}^{i}-S_{c}^{i}
$$

\footnotetext{
${ }^{1}$ standard summation convention is used, for the notation we refer again to Appendix A
} 
Besides the effects of bathymetry and friction (first two terms), the source includes also a geometrical force due to Earth curvature $S_{\gamma}^{i}$, and Coriolis term $S_{c}^{i}$. The geometric source arises from the space variation of the covariant basis vectors and reads

$$
S_{\gamma}^{i}=\Gamma_{j k}^{i} T^{j k}
$$

with $\Gamma_{j k}^{i}$ being the Christoffel symbols. The Coriolis term is associated to the rotation of the Earth, which plays an important role in ocean dynamics. It reads

$$
S_{c}^{i}=f G^{i j} \varepsilon_{j k} h u^{k}
$$

with the parameter $f=2 \Omega \sin \lambda$ with $\lambda$ the latitude and $\Omega$ the Earth rotation rate, always taken as $\Omega=7.292 \times 10^{-5} s^{-1}$ unless differently specified, and having denoted by $\varepsilon$ the 2 D Levi-Civita symbol.

The SWEs system can also be expressed in a compact vector form

$$
\begin{aligned}
& \frac{\partial \mathbf{u}}{\partial t}+\frac{1}{\sqrt{G}} \frac{\partial \mathrm{F}^{j}}{\partial x^{j}}+\mathcal{S}(\boldsymbol{x}, \mathbf{u})=0, \\
& \mathbf{u}=\left[\begin{array}{c}
h \\
h u^{i}
\end{array}\right], \mathrm{F}^{j}=\sqrt{G}\left[\begin{array}{c}
h u^{j} \\
T^{i j}
\end{array}\right] \\
& \mathcal{S}=\underbrace{\left[\begin{array}{c}
0 \\
G^{i j} g h \frac{\partial b}{\partial x^{j}}
\end{array}\right]}_{\mathcal{S}^{b}}+\underbrace{\left[\begin{array}{c}
0 \\
c_{F} h u^{i}
\end{array}\right]}_{\mathcal{S}^{f}}+\underbrace{\left[\begin{array}{c}
0 \\
\Gamma_{j k}^{i} T^{j k}
\end{array}\right]}_{\mathcal{S}^{\gamma}}-\underbrace{\left[\begin{array}{c}
0 \\
f G^{i j} \varepsilon_{j k} h u^{k}
\end{array}\right]}_{\mathcal{S}^{c}}
\end{aligned}
$$

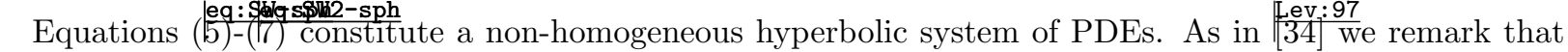
the flux vector depends explicitly on the position making the system non-autonomous.

\section{Arbitrary Lagrangian Eulerian framework on the sphere}

We now introduce an independent configuration $\mathcal{A}$, the Arbitrary Lagrangian Eulerian (ALE) configuration, which we assume to belong to a differentiable manifold (the sphere). For us $\mathcal{A}$ will be the configuration the mesh is attached to. $\mathcal{A}$ is composed of points $Q$ identified by the vector $\boldsymbol{x}$ which can be expressed in the local curvilinear coordinate system $\boldsymbol{x}(Q)=x^{i} \boldsymbol{g}_{i}$, with $i=1,2$. The initial or reference configuration of the continuous mesh is denoted by $\mathcal{A}_{0}$ and the reference position of the points is denoted by greek letters $\chi(\hat{Q})$. For this configuration we assign a local covariant vector basis $\left\{\gamma_{1}, \gamma_{2}\right\}$ and local chart $\left\{\chi^{1}, \chi^{2}\right\}$ of the tangent plane so that $\chi(\hat{Q})=\chi^{i} \boldsymbol{\gamma}_{i}$. As the mesh moves, also $\mathcal{A}$ does. We assume to be able to uniquely map the initial configuration to the reference one via the mapping:

$$
A: \mathcal{A}_{0} \rightarrow \mathcal{A} \quad \boldsymbol{x}=A(\chi, t)
$$


The function $A$ is assumed to be continuously differentiable, with a smooth Jacobian $\boldsymbol{J}_{A}=\frac{\partial \boldsymbol{x}}{\partial \boldsymbol{X}}$. Classical requirements on the Jacobian $J_{A}$ are invertibility $\exists J_{A}^{-1}$, and the positivity of its determinant $J_{A}=$

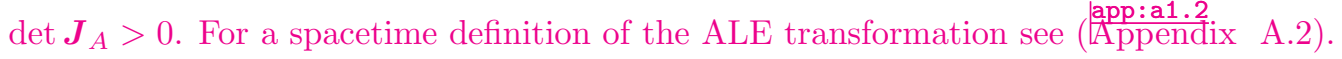

The fluid is endowed with various physical properties which may be represented by scalar, vector and tensor fields. These fields and their rate of change in time and space may be defined in the reference configuration or in the actual one. Here we choose the actual description: that is, in the following, all spatial derivatives are expressed with respect to the actual coordinates. The ALE time derivative of a scalar $\phi$ is its time rate of change measured by an observer which moves with the mesh point labeled with $\chi$, where $\chi(\hat{Q})$ is the particle's position in the reference configuration. We will shorten it $\left.\frac{\partial \phi}{\partial t}\right|_{\chi}$. The ALE/mesh velocity of a point $Q$ in the actual vector basis, $\boldsymbol{\sigma}(Q)=\sigma^{j} \boldsymbol{g}_{j}$ is calculated by applying the aforementioned definition of ALE derivative, to the position $\boldsymbol{x}(Q)$. Its components write

$$
\sigma^{j}=\left.\frac{\partial x^{j}}{\partial t}\right|_{\chi}
$$

Using chain rule, we can define relations between Eulerian and ALE time derivatives for these properties:

$$
\left.\frac{\partial \phi}{\partial t}\right|_{\chi}=\frac{\partial \phi}{\partial t}+\frac{\partial \phi}{\partial x^{j}} \sigma^{j}
$$

Finally, taking the ALE time derivative of an infinitesimal area on the sphere we obtain the Geometric Conservation Law on the manifold $\frac{\text { Tru: } 66}{35}:$

$$
\left.\frac{\partial}{\partial t}\right|_{\chi}\left(\sqrt{G} J_{A}\right)=J_{A} \frac{\partial}{\partial x^{j}}\left(\sqrt{G} \sigma^{j}\right)
$$

or, more compactly $\left.\partial_{t}\right|_{\chi}\left(\sqrt{G} J_{A}\right)-J_{A} \sqrt{G} \nabla \cdot \sigma=0$.

The GCL $\frac{\text { legi }}{111}$ is a is geometrical relation between the ALE Jacobian and the ALE velocity. One can

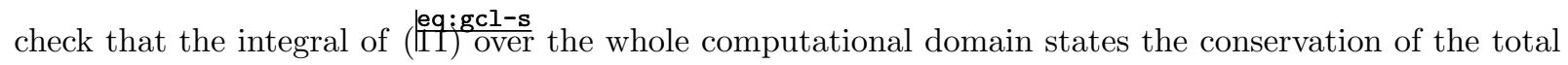
area. At a discrete level this corresponds to preserve the total area of the mesh during the simulation. For this reason in section 5.3 ise 5 we will discuss how to preserve also its discrete counterpart.

\section{ALE-SWEs on the sphere}

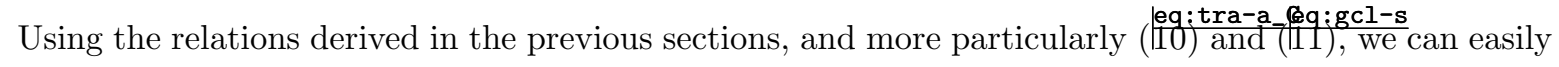
recast the SWEs (10.1):SW-s in an ALE form, obtaining the ALE-SWEs on a $2 \mathrm{D}$ manifold with respect to the 
actual parametric coordinates:

$$
\begin{array}{r}
\left.\frac{\partial \sqrt{G} J_{A} h}{\partial t}\right|_{\chi}+J_{A} \frac{\partial}{\partial x^{j}}\left(\sqrt{G} h u^{j}-\sqrt{G} h \sigma^{j}\right)=0 \\
\left.\frac{\partial \sqrt{G} J_{A} h u^{i}}{\partial t}\right|_{\chi}+J_{A} \frac{\partial}{\partial x^{j}}\left(\sqrt{G} T^{i j}-\sqrt{G} h u^{i} \sigma^{j}\right)+\sqrt{G} J_{A} S^{i}=0
\end{array}
$$

As before, the system can be also cast in compact vector form as

$$
\left.\frac{\partial}{\partial t}\right|_{\chi}\left(\sqrt{G} J_{A} \mathrm{u}\right)+J_{A} \frac{\partial}{\partial x^{j}}\left(\mathrm{~F}^{j}-\sqrt{G} \sigma^{j} \mathrm{u}\right)+\sqrt{G} J_{A} \mathcal{S}(\boldsymbol{x}, \mathrm{u})=0
$$

Finally, it is useful for the following to recall that the bathymetry, which does not depends explicitly on time $b=b(\boldsymbol{x})$, verifies the following identity:

$$
\left.\frac{\partial b}{\partial t}\right|_{\chi}=\frac{\partial b}{\partial x^{j}} \sigma^{j}
$$

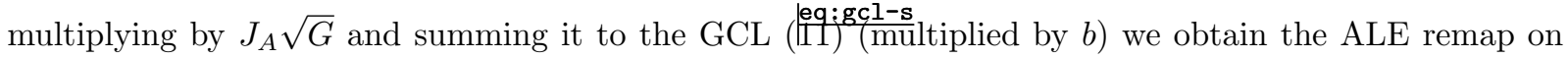
the $2 \mathrm{D}$ manifold

$$
\left.\frac{\partial \sqrt{G} J_{A} b}{\partial t}\right|_{\chi}-J_{A} \frac{\partial}{\partial x^{j}}\left(\sqrt{G} b \sigma^{j}\right)=0
$$

eq:b_ALE_remar

Please note that scalar, vector, tensor fields and spatial derivatives are written w.r.t. the actual coordinates

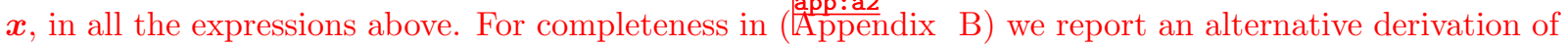
the governing equations $\frac{\text { ifj }}{\text { SW1-as }}$ with a spacetime approach.

\section{Discretization: parametrization and constraints}

In this section we discuss the basic aspects of the discretization. These involve the main geometrical notations as well as the form of the equation used for the discretization. We will introduce an appropriate form of the ALE-SWEs allowing to combine well-balancedness with geometric conservation. We then show how to embed discrete analogs of these constraints in a residual distribution setting.

\subsection{Unstructured grid representations: main notation}

Points on a manifold are represented by a proper parametrization through the use of geometrical mappings. In particular, when working in ALE formalism, a point $i$ will be expressed in the parametrization of the initial configuration by a vector $\chi_{i}=\left\{\chi^{1}, \chi^{2}\right\}_{i}$, while $\boldsymbol{x}_{i}=\left\{x^{1}, x^{2}\right\}_{i}$ will refer to the configuration attached to the mesh. Note that the discussion here is general, even though the numerical applications will only consider a latitude longitude parametrization of the sphere. This particular choice is known 
to introduce a singularity at the poles, which is nowadays well handled by meteorologists $\frac{\text { Wi1 }}{36}: 07$. Since we are interested in oceanic applications we circumvent this issue by adopting a simple fix. A patch-wise

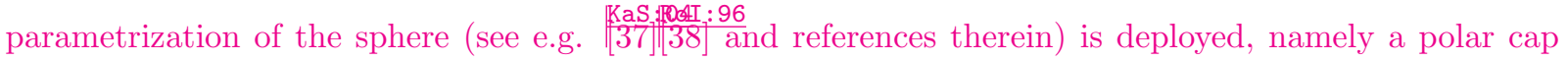
defined by a limiting latitude $\lambda_{\lim }$, in which we use a rotated reference system $\left(x^{\prime}, y^{\prime}, z^{\prime}\right)=(x, z,-y)$. To account for this "patching", a rotation operator needs to be applied when updating vectors in elements crossing the patch boundary. In our case, this applies both to physical velocities, as well as mesh nodes velocities and position. In all right hand side evaluations this is accounted for following 18 to which we refer for details.

We consider a discretization of $\mathcal{S}^{2}$ composed by non overlapping triangular elements $\mathcal{K}$. To each element $\mathcal{K}$ we can associate an element defined in the parametric space, which we denote by $K$. For each element, we define the spherical area and median dual cell of a node as

$$
|\mathcal{K}(t)|=\int_{K(t)} \sqrt{G} d \boldsymbol{x}, \quad\left|\mathcal{C}_{i}\right|=\sum_{K \in \mathcal{D}_{i}} \frac{|\mathcal{K}|}{3}
$$

eq: $s p-t r$

For moving meshes we also have:

$$
|\mathcal{K}(t)|=\int_{K_{0}} J_{A}(t) \sqrt{G} d \chi
$$

denoting by $K_{0}$ the element in the initial parametric space. Please note that, after the change of coordinates, we assume in the above expression that $\sqrt{G(\boldsymbol{x}(\boldsymbol{\chi}, t))}$. This duality of integral formulas via the ALE mapping applies to all integrals and will not be recalled explicitly unless necessary.

For a given mesh, we label by $\mathrm{h}$ the largest element diameter in the parameter space $M^{2}$. For a smooth enough initial mesh, and on a smooth manifold, we unduly assume that the number of elements $N_{K}$ verifies

$$
N_{K}|K| \leq c\left|M^{2}\right|
$$

eq:mesh-smootl

for some bounded constant. We also denote by $\mathrm{u}_{\mathrm{h}}$ the $P^{1}$ approximation of a quantity $\mathrm{u}$

$$
\mathrm{u}_{\mathrm{h}}(\boldsymbol{x})=\sum_{i} \mathrm{u}_{i} \varphi_{i}(\boldsymbol{x})
$$

having denoted by $\varphi_{i}$ the standard $P^{1}$ bases. We now define elemental contributions to the Galerkin mass matrix

$$
\left(m_{i j}^{G A L}\right)^{K}:=\int_{K} \varphi_{i} \varphi_{j} \sqrt{G}(\boldsymbol{x}) d \boldsymbol{x},
$$

eq:gal-mass

as well as to the lumped mass matrix

$$
\left(m_{i j}^{G A L}\right)^{L, K}:=\mathcal{K}_{i} \delta_{i j}, \quad \mathcal{K}_{i}:=\int_{K} \sqrt{G}(\boldsymbol{x}) \varphi_{i} d \boldsymbol{x}
$$




\subsection{Well-Balancedness and $G C L$ on the sphere}

For shallow water flows, a very important role is played by the lake-at-rest steady state:

$$
h \boldsymbol{u}=0, \quad h+b=\eta_{0}=\text { const }
$$

The exact preservation of this (initial) steady state at the discrete level is referred to as the C-property, or Well-Balancedness $\left[\begin{array}{l}\text { Bev: } 94 \\ 39\end{array}\right.$. In other words, well-balanced schemes provide a discrete analog of the balance (cf. equation (5):

$$
\frac{\partial \mathrm{F}^{j}}{\partial x^{j}}+\sqrt{G} \mathcal{S}=0 .
$$

Written in the form $\frac{\text { ieg } \mathrm{SW} 1 \text {-as }}{114}$, the ALE-SWEs require the simultaneous satisfaction of several compatibility constraints in order to devise well-balanced schemes. These constraints include:

1. well-balancedness for the Cartesian SWEs on fixed grids: $\partial_{x^{i}}\left(g h^{2} / 2\right)+g h \partial_{x^{i}} b=0, \forall i$;

2. a discrete analog of the GCL on moving grids $(110]^{g c 1-s}$

3. the ALE remap of the bathymetry leg_b_ALE_remap

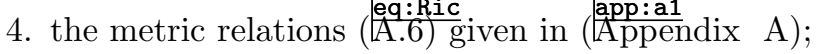

We will show in the following of the paper how we can embed exactly the first two constraints at a discrete

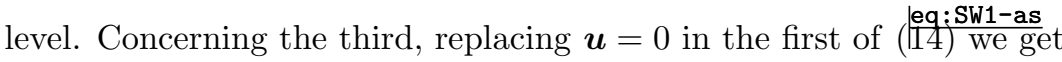

$$
\begin{aligned}
0 & =\left.\partial_{t}\left(\sqrt{G} J_{A} h\right)\right|_{\chi}-J_{A} \partial_{x^{j}}\left(\sqrt{G} \sigma^{j} h\right) \\
& =\left.\partial_{t}\left(\sqrt{G} J_{A} \eta\right)\right|_{\chi}-J_{A} \partial_{x^{j}}\left(\sqrt{G} \sigma^{j} \eta\right)-\left[\left.\partial_{t}\left(\sqrt{G} J_{A} b\right)\right|_{\chi}-J_{A} \partial_{x^{j}}\left(\sqrt{G} \sigma^{j} b\right)\right]
\end{aligned}
$$

If $\eta=\eta_{0}$ we see that a necessary condition for the lake-at-rest to be a solution for system $\frac{\text { i }}{14}$ SW1-as is to verify both the GCL, and the ALE remap of the bathymetry. Some authors [7hC: 13 have proposed to directly use a discretization of (1T) to evolve the topography on a moving mesh. The drawback of this approach is that it significantly modifies the bathymetry during the computation, which cannot be accepted in practical applications. For this reason, here we follow $\frac{A r R: 17}{1]}$ and modify $\left(\frac{\mid l a g}{14}\right)_{\text {by }}$ by solving instead the system obtained by adding to the first equation the ALE remap of the bathymetry:

$$
\left.\frac{\partial}{\partial t}\right|_{\chi}\left(\sqrt{G} J_{A} \mathrm{w}\right)+J_{A} \frac{\partial}{\partial x^{j}}\left(\mathrm{~F}^{j}-\sqrt{G} \sigma^{j} \mathrm{w}\right)+\sqrt{G} J_{A} \mathcal{S}(\boldsymbol{x}, \mathrm{u})=0, \mathrm{w}=\left[\begin{array}{c}
\eta \\
h \boldsymbol{u}
\end{array}\right]
$$

Note that, with this notation, we have $\mathrm{F}^{j}(\mathrm{u})=\mathrm{F}^{j}(\mathrm{w} ; b)$, as the bathymetry is required to evaluate the flux starting from the w array. Concerning now the fourth requirement, we consider the momentum balance 


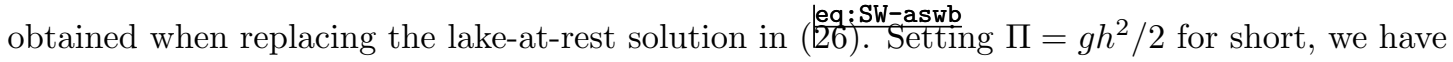

$$
\begin{aligned}
0 & =J_{A}\left\{\partial_{x^{j}}\left(\sqrt{G} G^{i j} \Pi\right)+\sqrt{G} \Gamma_{j k}^{i} G^{j k} \Pi+g h \sqrt{G} G^{i j} \partial_{x^{j}} b\right\} \\
& =J_{A}\left\{\sqrt{G} G^{i j}\left(g h \partial_{x^{j}} b+\partial_{x^{j}} \Pi\right)+\Pi\left[\partial_{x^{j}}\left(\sqrt{G} G^{i j}\right)+\sqrt{G} \Gamma_{j k}^{i} G^{j k}\right]\right\}
\end{aligned}
$$

The first group in parentheses vanishes if the first property (well-balancedness for Cartesian SWEs on fixed grids) is satisfied. The remainder is nothing else that the combination of the two metric relations.

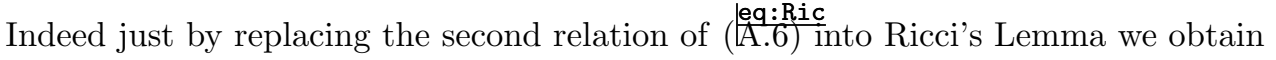

$$
\begin{aligned}
0 & =\sqrt{G}\left(\frac{\partial G^{i j}}{\partial x^{j}}+G^{i j} \Gamma_{m j}^{m}+\Gamma_{j m}^{i} G^{m j}\right) \\
& =\sqrt{G} \frac{\partial G^{i j}}{\partial x^{j}}+G^{i j} \frac{\partial \sqrt{G}}{\partial x^{j}}+\sqrt{G} \Gamma_{j m}^{i} G^{m j}=\frac{\partial \sqrt{G} G^{i j}}{\partial x^{j}}+\sqrt{G} \Gamma_{j m}^{i} G^{m j}
\end{aligned}
$$

The symmetry of the Christoffel symbols $\frac{\text { Nou }}{411}$ allows to recover exactly the last expression in parentheses in [27]. The above relation is thus necessary for the lake-at-rest solution to be verified. At the discrete level, the easiest way to make sure this condition is embedded is to combine high order quadrature with the direct use of the analytical formulas. Even though low quadrature errors can be obtained with relatively few quadrature points, due to the smoothness of the functions involved, in this way the C-property would be satisfied within this quadrature error. Also, compared to the Cartesian case, the necessity of using higher order quadrature would still give a considerable overhead. For this reason we have chosen to embed the metric relations analytically. Whenever possible we discretize the momentum balance in the following form:

$$
\begin{aligned}
\left.\frac{\partial}{\partial t}\right|_{\chi}\left(\sqrt{G} J_{A} h u^{i}\right) & +J_{A} \frac{\partial}{\partial x^{j}}\left(\sqrt{G} h u^{i}\left(u^{j}-\sigma^{j}\right)\right)+J_{A} \sqrt{G} G^{i j}\left(\frac{\partial}{\partial x^{j}}\left(g h^{2} / 2\right)+g h \frac{\partial}{\partial x^{j}} b\right) \\
& +J_{A} \sqrt{G} S^{i}=0 .
\end{aligned}
$$

For later use in the paper, we introduce the short notation

$$
\left.\frac{\partial\left(J_{A} \sqrt{G} \mathrm{w}\right)}{\partial t}\right|_{\chi}+J_{A} \mathcal{R}(\mathrm{w}, b)=0
$$

for the system obtained with the first in $[26]$, plus $[29]$.

\section{3. $D G C L$ on the sphere}

We consider now the satisfaction of the discrete analog of the GCL an a 2D manifold $\left(\frac{\text { lifjecl-s }}{11 .}\right.$ As already said, differently from the Cartesian case, a uniform flow is not an exact solution of (10 SW1-as embedded with the GCL $[11]$, even for constant bathymetries and in absence of friction and Coriolis force. As a consequence we cannot define the DGCL through the original characterization of [28]: 79 i.e. the preservation of a uniform 
flow. On the sphere, we could replace the preservation of uniform flow with some other particular cases, as e.g. the preservation of zonal or meridional flows. However, the most relevant characterization of the GCL for the applications of this paper is the one we have introduced in the previous section, namely the satisfaction of the lake-at-rest state.

It is thus in the perspective of obtaining well balanced schemes on moving grids that we consider the issue of mimicking (110 at the discrete level. As we will shortly see, the main issue is properly defining the components of the mesh velocity $\sigma^{j}$ along certain directions, as well as an approximation of the divergence operator $\partial_{x^{j}}\left(\sqrt{G} \sigma^{j}\right)$. The DGCL closure used in previous works by the authors ArR: 14, ArR: 17 , in and inspired by LeF: 26, GuF:00 recover naturally an element-by-element DGCL when integrating the divergence of the displacements over the half time step average element $K^{n+1 / 2}=\left(K^{n+1}+K^{n}\right) / 2$. Due to the presence of the coefficient $\sqrt{G}$, the extension of this approach is not at all straightforward. We have thus chosen to use a generalization of the closure originally proposed in $\frac{\mathrm{May}: 06}{42}$.

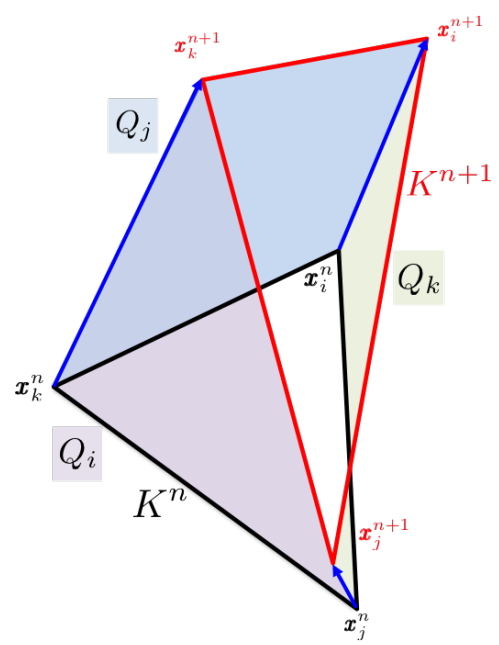

Figure 1: Swept area decomposition of area variations for mesh element $K . \quad$ fig:swept-area

To describe this closure, we consider an element $\mathcal{K}$ of the mesh, or equivalently its projection in the parametric space $K$. We study the discrete approximation of the area variation

$$
\left|\mathcal{K}^{n+1}\right|-\left|\mathcal{K}^{n}\right|=\left[\int_{K_{0}} J_{A} \sqrt{G} d \chi\right]^{n+1}-\left[\int_{K_{0}} J_{A} \sqrt{G} d \chi\right]^{n}
$$

We decompose this variation in components associated to the area swept by each face, as illustrated in figure 1 if. We have in particular

$$
\left|\mathcal{K}^{n+1}\right|-\left|\mathcal{K}^{n}\right|=\Delta t \sum_{j \in K} v_{f_{j}}
$$




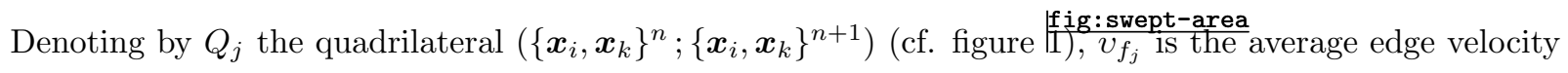
tangent to the manifold and normal to the edge defined by

$$
v_{f_{j}}:=\frac{1}{\Delta t} \int_{Q_{j}} \sqrt{G} d \boldsymbol{x}
$$

eq:ivc-s

In practice, the integral is computed by a high order quadrature formula

$$
\int_{Q_{j}} \sqrt{G} d \boldsymbol{x}=\left|Q_{j}\right| \sum_{\boldsymbol{x}_{q}} \omega_{q} \sqrt{G}\left(\boldsymbol{x}_{q}\right)
$$

where the quadrature grid is built simply by tensorizing 1D quadrature points over each edge, and the signed volume $\left|Q_{j}\right|$ is evaluated using the surveyor's (or shoelace) area formula $\mid$ shoelace

To discretize the ALE-SWEs we now need to provide a discrete approximation of the curvilinear divergence appearing in the GCL. For the purposes of the paper we would like this definition to be compatible with the following relations

$$
\begin{aligned}
\mathcal{K}^{n+1}-\mathcal{K}^{n} & =\left.\int_{t^{n}}^{t^{n+1}} \int_{K_{0}} J_{A} \frac{\partial}{\partial x^{j}}\left(\sqrt{G} \sigma^{j}\right)\right|_{K} d \chi d t \\
\mathcal{K}_{i}^{n+1}-\mathcal{K}_{i}^{n} & =\left.\int_{t^{n}}^{t^{n+1}} \int_{K_{0}} J_{A} \varphi_{i} \frac{\partial}{\partial x^{j}}\left(\sqrt{G} \sigma^{j}\right)\right|_{K} d \chi d t \\
\int_{K_{0}} J_{A}^{n+1} \sqrt{G} \varphi_{i} \mathrm{w}_{\mathrm{h}}-\int_{K_{0}} J_{A}^{n} \sqrt{G} \varphi_{i} \mathrm{w}_{\mathrm{h}} & =\left.\int_{t^{n}}^{t^{n+1}} \int_{K_{0}} J_{A} \varphi_{i} \frac{\partial}{\partial x^{j}}\left(\sqrt{G} \sigma^{j}\right)\right|_{K} \mathrm{w}_{\mathrm{h}} d \boldsymbol{\chi} d t
\end{aligned}
$$

with $w_{h}$ the approximation of the same data on the different meshes associated to the mappings with Jacobians $J_{A}^{n+1}$ and $J_{A}^{n}$. The first relation is the spacetime integral of the GCL while the second and the third relations are useful to derive a consistent finite element method, and mass lumping. The practical evaluation of each of the integrals on the left hand side plays here a fundamental role in our ability to satisfy exactly the three relations above. We have the following characterization.

Proposition 1. (Approximate quadrature on the manifold and DGCL) Consider a one point quadrature approximating the integral over a time step as

$$
\int_{t^{n}}^{t^{n+1}} \int_{K_{0}} J_{A} f(\boldsymbol{\chi}) d \chi d t=\Delta t \int_{K_{0}} \bar{J}_{A} f(\boldsymbol{\chi}) d \chi d t=\Delta t \int_{\bar{K}} f(\overline{\boldsymbol{x}}) d \overline{\boldsymbol{x}} d t
$$

and the constant-by-element approximation of the divergence

$$
\left.\frac{\partial}{\partial x^{j}}\left(\sqrt{G} \sigma^{j}\right)\right|_{\bar{K}}:=\frac{1}{|\overline{\mathcal{K}}|} \sum_{j \in \bar{K}} v_{f_{j}}
$$




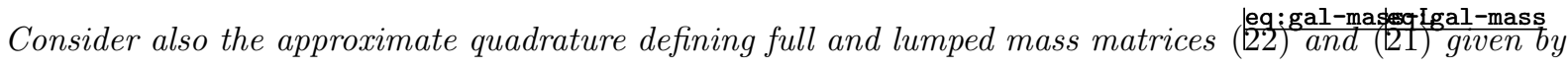

$$
\left(m_{i j}^{G A L}\right)^{K} \approx \frac{|\mathcal{K}|}{3} \frac{1+\delta_{i j}}{4}, \quad\left(m_{i j}^{G A L}\right)^{L, K}=\mathcal{K}_{i} \delta_{i j} \approx \frac{|\mathcal{K}|}{3} \delta_{i j} .
$$

eq:gal-mass-d

We have the following properties:

1. Relations (34), (35) and (3j) are verified exactly;

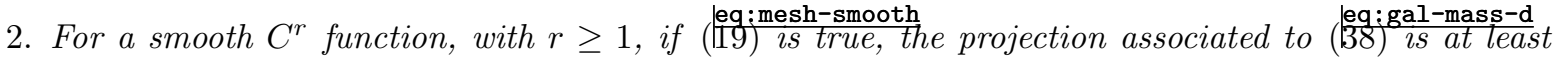
second order accurate.

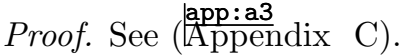

Remark 1. (Higher order quadrature in mass matrices). The approximate quadrature (3ิ8) is only required to embed exactly the GCL in the scheme. In absence of mesh movement, higher order (or exact) quadrature can be used to define the mass matrix entries, which will differ from $\frac{\text { (3) gal-mass-d }}{38}$ within the quadrature error just proved. In practice we have seen no advantage in using more accurate quadrature in these formulas. For completeness, the modifications necessary to account (on fixed meshes) for the exact evaluation of the mass matrices for Residual Distribution and stabilized finite elements are provided in (APppendix D).

Note that in practice, when using the sphere with lat-lon parametrization, the gradient of $\sqrt{G}$ appearing in the error estimate in the appendix is given by $R^{2} \sin \lambda$ with $\lambda$ the latitude, which is clearly bounded.

Finally, note that the choice of $\bar{J}_{A}$ is arbitrary and has no impact on the satisfaction of the DGCL. For accuracy reasons, here we have kept a mid-point approximation $\bar{J}_{A}=\left(J_{A}^{n}+J_{A}^{n+1}\right) / 2$. This means that, most geometrical quantities are evaluated as averages on the elements $\mathcal{K}^{n+1}$ and $\mathcal{K}^{n}$. This is implicitly assumed in all the formulas which will follow. For simplicity, in the text we will drop the superscript altogether, unless necessary in the formula.

\section{ALE Residual Distribution on the sphere}

A first extension of Residual Distribution (RD) to general covariant coordinates has been proposed by Ros: 13 The work of the reference is limited to steady state computations, with linear schemes and without bathymetry. This paper presents a general second order and well balanced formulation allowing time dependent computations on moving meshes. The construction proposed here is a generalization of the work done in Cartesian coordinates in $\left[\begin{array}{l}A r R: 17 \\ {[1]}\end{array}\right.$ for a thorough discussion of the properties of residual distribution. 
The RD discretization of unsteady problems can be described starting with an analogy with stabilized continuous finite elements. Given a triangulation of the parametric domain $S^{2}$, to solve $\mathcal{L}\left(\mathrm{w}_{h}\right)=0$, one can formally recast a $\mathrm{RD}$ scheme as

$$
\int_{S^{2}} w_{i} \mathcal{L}\left(\mathrm{w}_{h}\right) d \boldsymbol{\chi}=0
$$

where $w_{i}=\varphi_{i}+\gamma_{i}$ is a Petrov-Galerkin test function, with $\gamma_{i}$ an appropriately defined bubble function. For consistency, on each element $K$

$$
\sum_{j \in K} w_{j}=1
$$

eq:rd-consist

which implies $\sum_{j} \gamma_{j}=0$. We define the element residual associated to [26] as the area integral over the element of the full differential operator:

$$
\Phi^{K}=\int_{K_{0}} \mathcal{L}\left(\mathrm{w}_{h}\right) d \boldsymbol{\chi}
$$

The consistency allows to define the distributed residuals

$$
\Phi_{i}^{K}:=\int_{K_{0}} w_{i} \mathcal{L}\left(\mathrm{w}_{h}\right) d \chi
$$

eq:rd-splito

which verify the consistency condition

$$
\sum_{j \in K} \Phi_{j}^{K}=\Phi^{K}
$$

In actual computations, the main component of the distributed residuals is evaluated by means of distribution matrices $\boldsymbol{\beta}_{i}^{K}$ which also verify consistency

$$
\sum_{j \in K} \boldsymbol{\beta}_{j}^{K}=\mathrm{I} .
$$

The bridge with finite elements is completed by the requirement

$$
\boldsymbol{\beta}_{i}^{K}=\frac{1}{\left|K_{0}\right|} \int_{K_{0}} w_{i} d \boldsymbol{\chi}=\bar{w}_{i}^{K} .
$$

which leads to form of the distribution used in practice:

$$
\Phi_{i}^{K}=\boldsymbol{\beta}_{i}^{K} \Phi^{K}+\int_{K_{0}}\left(w_{i}-\bar{w}_{i}^{K}\right) \mathcal{L}\left(\mathrm{w}_{h}\right) d \boldsymbol{\chi}
$$


In its final form, the scheme evolves nodal values of the state vector w with a two-step procedure, in which the new solution value is obtained from:

$$
\left|\mathcal{C}_{i}^{n+1}\right|\left(\mathrm{w}_{i}^{n+1}-\mathrm{w}_{i}^{*}\right)=-\Delta t \sum_{K \ni i} \Phi_{i}^{K}
$$

where the nodal residuals $\Phi_{i}^{K}$ are a splitting of the element residual

$$
\sum_{j \in K} \Phi_{j}^{K}=\Phi^{K}=\frac{1}{\Delta t} \int_{K_{0}}\left(J_{A}^{n+1} \sqrt{G} \mathrm{w}_{\mathrm{h}}^{*}-J_{A}^{n} \sqrt{G} \mathrm{w}_{\mathrm{h}}^{n}\right) d \chi+\frac{1}{2} \Phi^{K}\left(\mathrm{w}_{\mathrm{h}}^{*}, b_{\mathrm{h}}^{n+1}\right)+\frac{1}{2} \Phi^{K}\left(\mathrm{w}_{\mathrm{h}}^{n}, b_{\mathrm{h}}^{n}\right)
$$

with $\Phi^{K}(\mathrm{w}, b)$ the fluctuation (the area integral over the element $K$ of only advective and source terms)

$$
\begin{aligned}
\Phi^{K}=\oint_{\partial K} \sqrt{G}\left[\begin{array}{c}
\left(h u^{j}-\eta \sigma^{j}\right) \\
h u^{i}\left(u^{j}-\sigma^{j}\right)
\end{array}\right]_{\mathrm{h}} n^{j} d s+\int_{K} \sqrt{G}\left[\begin{array}{c}
0 \\
G^{i j}\left(\partial_{x^{j}}\left(g h^{2} / 2\right)+g h \partial_{x^{j}} b\right)
\end{array}\right]_{\mathrm{h}} d \boldsymbol{x} \\
+\int_{K} \sqrt{G}\left[\begin{array}{c}
0 \\
\mathcal{S}^{f}+\mathcal{S}^{\gamma}+\mathcal{S}^{c}
\end{array}\right]_{\mathrm{h}} d \boldsymbol{x}
\end{aligned}
$$

Please note that the fluctuation integral is evaluated with respect to the actual parametric coordinates after using $d \boldsymbol{x}=\bar{J}_{A} \boldsymbol{d} \boldsymbol{\chi}$. The predicted nodal values $\mathrm{w}_{i}^{*}$ are computed from

$$
\left|\mathcal{C}_{i}^{n+1}\right|\left(\mathrm{w}_{i}^{*}-\mathrm{w}_{i}^{n}\right)=-\Delta t \sum_{K \ni i} \tilde{\Phi}_{i}^{K}\left(\mathrm{w}_{\mathrm{h}}^{n}, b_{\mathrm{h}}^{n}\right)
$$

RC-pred

with the $\tilde{\Phi}_{i}^{K}$ defining a splitting of the geometrically non-conservative fluctuation (cf. $\left.\begin{array}{l}\text { App:as } \\ \text { Apendix D }\end{array}\right)$

$$
\begin{aligned}
\sum_{j \in K} \tilde{\boldsymbol{\Phi}}_{j}^{K}=\tilde{\boldsymbol{\Phi}}^{K} & =\oint_{\partial K} \sqrt{G}\left[\begin{array}{c}
h u^{j} \\
h u^{i} u^{j}
\end{array}\right]_{\mathrm{h}} n^{j} d s+\int_{K} \sqrt{G}\left[\begin{array}{c}
0 \\
G^{i j}\left(\partial_{x^{j}}\left(g h^{2} / 2\right)+g h \partial_{x^{j}} b\right)
\end{array}\right]_{\mathrm{h}} d \boldsymbol{x} \\
& -\int_{K} \sqrt{G} \sigma^{j} \frac{\partial \mathrm{w}_{\mathrm{h}}}{\partial x^{j}} d \boldsymbol{x}+\int_{K} \sqrt{G}\left[\begin{array}{c}
0 \\
\mathcal{S}^{f}+\mathcal{S}^{\gamma}+\mathcal{S}^{c}
\end{array}\right]_{\mathrm{h}} d \boldsymbol{x}
\end{aligned}
$$

\section{RC-corr}




\subsection{Evaluation of the integrals}

To begin with, unless explicitly specified, in all quadrature formulas we have used analytical expressions for both the Jacobian $\sqrt{G}$, and the metric tensor $G^{i j}$. At the quadrature points, the mass flux components $\left(h u^{i}\right)_{\mathrm{h}}$ are evaluated simply using the $P 1$ expansion, while velocity components are obtained from the interpolated values of momentum $\left(h u^{i}\right)_{\mathrm{h}}$ and depth $h_{\mathrm{h}}$. The wet/dry region is handled as discussed in RiB:09, Ric: 15 , 11]. Consistently with the approximation (38) of Proposition 1, volume integrals of the unknown are computed as

$$
\int_{K_{0}} J_{A}^{n+1} \sqrt{G} \mathrm{w}_{\mathrm{h}} d \chi=\sum_{j \in K}\left|\mathcal{K}_{j}^{n+1}\right| \mathrm{w}_{j}=\frac{\left|\mathcal{K}^{n+1}\right|}{3} \sum_{j \in K} \mathrm{w}_{j}
$$

In all boundary integrals, scaled constant-by-face values of the normal mesh velocity $\sigma^{j} n^{j}$ are computed

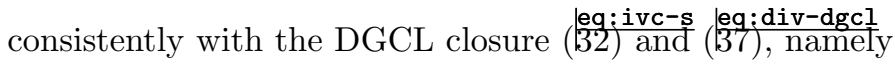

$$
\left(\sqrt{G} \sigma^{j} n^{j}\right)_{f}:=v_{f}
$$

Concerning the non-conservative ALE term in $\tilde{\Phi}^{K}$, this has been evaluated as

$$
\int_{K} \sqrt{G} \sigma^{j} \frac{\partial \mathrm{w}_{\mathrm{h}}}{\partial x^{j}} d \boldsymbol{x}=\oint_{\partial K} \sqrt{G} \mathrm{w}_{\mathrm{h}} \sigma^{j} n^{j} d s-\int_{K} \mathrm{w}_{\mathrm{h}} \frac{\partial \sqrt{G} \sigma^{j}}{\partial x^{j}} d \boldsymbol{x}
$$

where the face values (533) are used in the boundary integral, while the element integral is evaluated by means of the constant divergence of the DGCL $\left[\begin{array}{ll}37 \\ 37\end{array}\right]$ :

$$
\int_{K} \sqrt{G} \sigma^{j} \frac{\partial \mathrm{w}_{\mathrm{h}}}{\partial x^{j}} d \boldsymbol{x}=\sum_{i \in K} v_{f_{i}} \mathrm{w}_{f_{i}}-\mathrm{w}_{K} \frac{\left|\mathcal{K}^{n+1}\right|-\left|\mathcal{K}^{n}\right|}{\Delta t}=\sum_{i \in K} v_{f_{i}}\left(\mathrm{w}_{f_{i}}-\mathrm{w}_{K}\right)
$$

where the average values $\mathrm{w}_{f}$ and $\mathrm{w}_{K}$ are simple arithmetic averages over the faces and over the element.

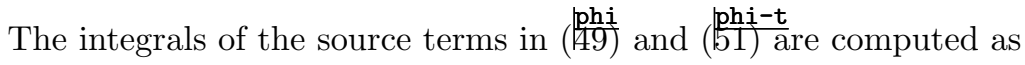

$$
\int_{K} \sqrt{G} \mathcal{S} d x \approx \frac{|\mathcal{K}|}{3} \sum_{j \in K} \mathcal{S}_{j}
$$

Finally, the hydrostatic term is computed as

$$
\begin{aligned}
& \int_{K} \sqrt{G} G^{i j}\left(\partial_{x^{j}}\left(g h_{\mathrm{h}}^{2} / 2\right)+g h_{\mathrm{h}} \partial_{x^{j}} b_{\mathrm{h}}\right) d \boldsymbol{x}=\int_{K} \sqrt{G} G^{i j} g h_{\mathrm{h}}(\overbrace{\partial_{x^{j}} h_{\mathrm{h}}+\partial_{x^{j}} b_{\mathrm{h}}}^{\partial_{x^{j}} \eta_{\mathrm{h}}}) d \boldsymbol{x} \\
&=\left.\left.\left(\int_{K} \sqrt{G} G^{i j} g h_{\mathrm{h}} d \boldsymbol{x}\right) \partial_{x^{j}} \eta_{\mathrm{h}}\right|_{K} \approx|K|\left(\sum_{q} \omega_{q} \sqrt{G} G^{i j}\left(\boldsymbol{x}_{q}\right) g h_{\mathrm{h}}\left(\boldsymbol{x}_{q}\right)\right) \partial_{x^{j}} \eta_{\mathrm{h}}\right|_{K}
\end{aligned}
$$

Several (second or higher order) quadrature formulas have been tested for the term in parentheses without any visible impact on the results.

6.3. Distribution of the residual and main properties

In RD schemes stability and accuracy properties depends on how the residual is distributed. The computation of the split residuals for the two RK steps $\Phi_{i}^{K}$ and $\tilde{\Phi}_{i}^{K}$ is performed following exactly the 
steps discussed in $\frac{\text { Ric: } 15}{11}$. In particular, following the construction in the reference, the residual splitting

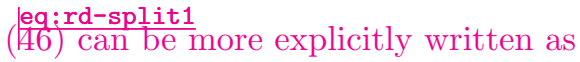

$$
\Phi_{i}^{K}=\boldsymbol{\beta}_{i}^{K} \Phi^{K}+\delta\left(\mathrm{w}_{\mathrm{h}}\right) \boldsymbol{\beta}_{i}^{\mathrm{SD}} \Phi^{K}+\delta\left(\mathrm{w}_{\mathrm{h}}\right) \sum_{j \in K} m_{i j}^{*} \frac{\mathrm{w}_{j}^{*}-\mathrm{w}_{j}^{n}}{\Delta t}
$$

In the above expression, the first term is the consistent one, namely the one satisfying $\sum_{j} \boldsymbol{\beta}_{j}^{K}=1$. The

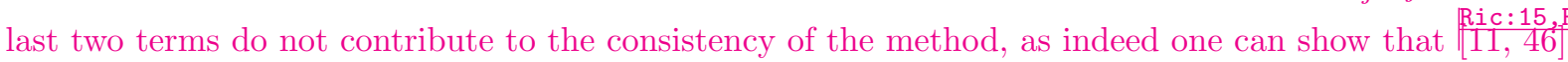

$$
\sum_{j \in K} \boldsymbol{\beta}_{j \in K}^{\mathrm{SD}} \Phi^{K}+\sum_{j \in K} \sum_{k \in K} m_{j k}^{*} \frac{\mathrm{w}_{k}^{*}-\mathrm{w}_{k}^{n}}{\Delta t}=0
$$

These additional terms are essentially stabilization terms allowing to avoid spurious modes appearing in

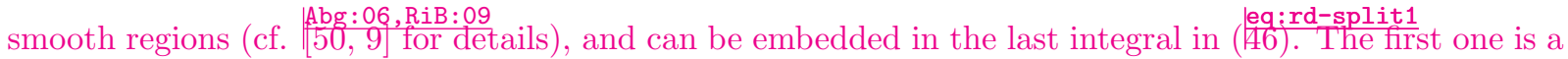
streamline upwind type stabilization, the second is a correction to a mass-lumping operator. These terms are in practice evaluated exactly as discussed in $\frac{A b \sigma}{50}, \frac{06}{9}, \frac{R i B}{11}$, 09 , Ric: 15 which we refer for details. Concerning the first term, as in $\frac{R i c: 15}{11}$ it is a distribution based on a blending, via the smoothness sensor $\delta\left(w_{h}\right)$, between a central splitting and a limited nonlinear splitting. The latter is obtained using a standard procedure in RD schemes involving the application of a bounded and sign-preserving mapping to a first order and

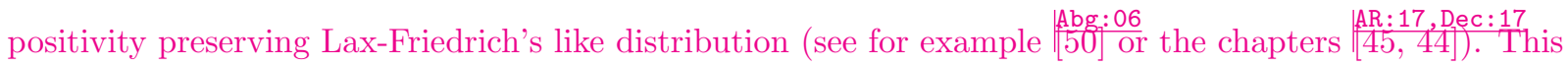
allows to combine formal second order of accuracy with depth non-negativity preservation.

Finally note that the distribution functions for the predictor step $(50)$ are easily deduced from $(58)$ setting $\mathrm{w}^{*}=\mathrm{w}^{n}$, and replacing $\Phi^{K}$ by $\tilde{\Phi}^{K}$.

Remark 2. (Metric terms treatment, accuracy, stability). It is worth noting that the scheme is formally identical to the one obtained in the planar case. The treatment of the geometric source term boils down to the modification of the cell residual. This is a substantial simplification when conceiving and coding the scheme compared to other methods (e.g Finite Volume).

Concerning the properties of the final discretization, the two main tools of analysis are a consistency property w.r.t. a variational form evaluated for smooth solutions, and a positivity preserving property

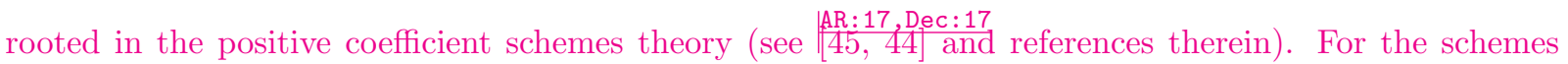
used here, high order of accuracy is formally ensured by the boundedness of the distribution matrices, and more generally of the test functions $\left\{w_{j}\right\}_{j \in K}$ in 46 . For the analysis of the depth positivity-preservation property of this Lax-Friedrich's-type scheme (as well as of the one obtained applying the limiter), the interested reader can refer to $\frac{\text { Ric: } 15}{11}$ for the Eulerian case. In the ALE case, an additional limiting step is 
required, as proposed in $\frac{a r R: 17}{1}$. This is to control the mismatch between the update in the mass equation, performed in terms of $\eta$, and the one of the bathymetry based on at and not on the ALE remap.

\subsubsection{Well-balancedness, DGCL and mass conservation}

The first property we can easily show is the following.

Proposition 2. (DGCL and well-balanced on the sphere) For any given mesh displacement on the

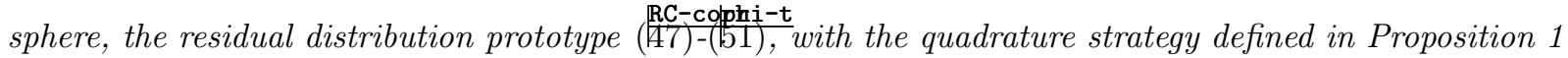
and by (55) and (5) , and with the distribution functions (58) verifies the C-property on moving and fixed meshes, as well as for Cartesian and curvilinear coordinates.

Proof. The proof is obtained by applying the scheme to the initial solution $\mathrm{w}^{0}=\left[\eta_{0}, 0,0\right]^{t}$. With

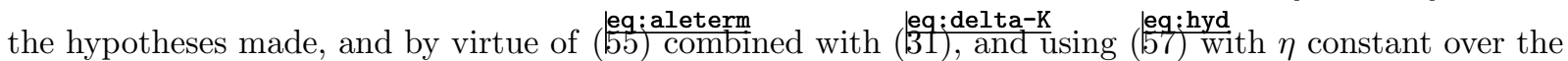
mesh, we deduce immediately that $\tilde{\Phi}^{K}=0$, thus $\tilde{\Phi}_{i}^{K}=0$, and $\mathrm{w}_{i}^{*}=\mathrm{w}_{i}^{0}$.

In the corrector step the element residual, evaluated with constant $\eta$ and invoking $\left(\frac{\log }{57}\right)$, reduces to

$$
\Phi^{K}=\frac{|\mathcal{K}|^{n+1}-|\mathcal{K}|^{n}}{\Delta t} \mathrm{w}^{0}-\sum_{j \in K} v_{f_{j}} \mathrm{w}^{0}=0
$$

due to the definition of $v_{f}$. Combined with the previous results and this implies $\mathrm{w}^{n+1}=\mathrm{w}^{0}$.

This proposition corroborates the choices made in the previous sections concerning the form of the SWEs. As already mentioned, however, the choice of $w$ as a main unknown has a drawback concerning

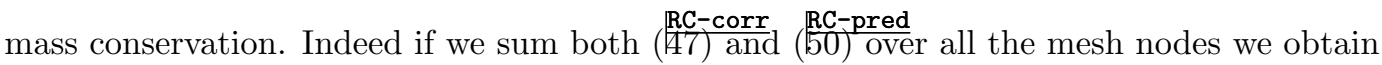

$$
\sum_{i}\left|\mathcal{C}_{i}^{n+1}\right| \mathrm{w}_{i}^{n+1}-\sum_{i}\left|\mathcal{C}_{i}^{n+1}\right| \mathrm{w}_{i}^{*}=-\Delta t \sum_{i} \sum_{K \ni i} \Phi_{i}^{K}=-\Delta t \sum_{K} \sum_{i \in K} \Phi_{i}^{K}=-\Delta t \sum_{K} \Phi^{K}
$$

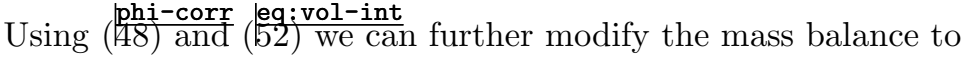

$$
\sum_{i}\left|\mathcal{C}_{i}^{n+1}\right| \mathrm{w}_{i}^{n+1}-\sum_{i}\left|\mathcal{C}_{i}^{n}\right| \mathrm{w}_{i}^{n}=-\frac{\Delta t}{2} \sum_{K} \Phi^{K}\left(\mathrm{w}_{\mathrm{h}}^{n}, b_{\mathrm{h}}^{n}\right)-\frac{\Delta t}{2} \sum_{K} \Phi^{K}\left(\mathrm{w}_{\mathrm{h}}^{*}, b_{\mathrm{h}}^{n+1}\right)
$$

If we only consider the mass equation, the right hand side gives

$$
-\frac{\Delta t}{2}\left[\sum_{K}\left(\oint_{\partial K} \sqrt{G}\left(h u^{j}-\eta \sigma^{j}\right)_{\mathrm{h}}^{n} n^{j}+\oint_{\partial K} \sqrt{G}\left(h u^{j}-\eta \sigma^{j}\right)_{\mathrm{h}}^{*} n^{j}\right)\right]=0
$$

face integrals of neighbor elements canceling each other. As a consequence we end with

$$
\sum_{i}\left|\mathcal{C}_{i}^{n+1}\right| h_{i}^{n+1}=\sum_{i}\left|\mathcal{C}_{i}^{n}\right| h_{i}^{n}-\left(\sum_{i}\left|\mathcal{C}_{i}^{n+1}\right| b_{i}^{n+1}-\sum_{i}\left|\mathcal{C}_{i}^{n}\right| b_{i}^{n}\right)
$$

mass-error 
As remarked in $\frac{A r R: 17}{1]}$ the error in total mas conservation is related to the error in satisfying a discrete ALE remap of the bed. To minimize the mass error, we use here the same technique proposed in $\mid A r R: 17$. We set:

$$
\left|\mathcal{C}_{i}^{n+1}\right| b_{i}^{n+1}:=\int_{C_{i}^{0}} J_{A}^{n+1} \sqrt{G} \hat{b}(\chi) d \chi
$$

where $\hat{b}(\chi)$ is a high resolution (analytical, or reconstructed using a finer mesh) description of the topography. We then evaluate the last integral using a higher order quadrature as done in $\frac{\operatorname{Ar} R: 17}{1}$. In particular, if the quadrature is of order $\kappa$ we have, considering a smooth enough manifold:

$$
\sum_{i}\left|\mathcal{C}_{i}^{n+1}\right| b_{i}^{n+1}=\sum_{i} \int_{C_{i}} J_{A}^{n+1} \sqrt{G} \hat{b}(\chi) d \chi+\sum_{i} \mathcal{O}\left(\mathrm{h}^{\kappa+3}\right)=\int_{M^{2}} \sqrt{G} b(\boldsymbol{x}) d \boldsymbol{x}+\mathcal{O}\left(\mathrm{h}^{\kappa+1}\right)
$$

where the $\mathcal{O}\left(\mathrm{h}^{\kappa+3}\right)$ is the local error modeled as a polynomial of degree (at least) $\kappa+1$, readily obtained using e.g. a truncated Taylor series, and accounting for the fact that $|\mathcal{K}|=\mathcal{O}\left(\mathrm{h}^{2}\right)$. The second equality uses 190 . Note now that the first term in the final expression is actually time independent as long as $M^{2}=M_{0}^{2}$, which is in practice the case. Since also the integrand is a time independent quantity, we have

$$
\left|\sum_{i}\right| \mathcal{C}_{i}^{n+1}\left|b_{i}^{n+1}-\sum_{i}\right| \mathcal{C}_{i}^{n}\left|b_{i}^{n}\right|=\mathcal{O}\left(\mathrm{h}^{\kappa+1}\right)
$$

254 255

sec:mmpde

which combined with $\frac{\text { mass-error }}{64}$ shows that we can conserve $h$ within an arbitrary accuracy.

\section{Adaptive mesh movement strategy}

The mesh adaptation technique used here is based on an error dependent mesh movement. The main principle is to define a mapping $A(\chi, t)$ from a reference/initial configuration, described by the coordinate $\boldsymbol{\chi}$, to a physical mesh described by coordinate $\boldsymbol{x}$, such that the error associated to a certain density is equi-distributed. The error density is often referred to as a monitor function $m(\boldsymbol{x})$ PeB: 73 . The differential form of this principle is referred to as Moving Mesh Partial Differential Equation or MMPDE for short. The simplest method in the multidimensional case is the elliptic generator based on the MMPDE:

$$
\frac{\partial}{\partial \chi^{i}}\left(m \frac{\partial x^{\alpha}}{\partial \chi^{i}}\right)=0 \quad \alpha=1,2
$$

This PDE is the Euler-Lagrange equation corresponding to the minimization of the energy functional $\mid \frac{\mathrm{CeH}}{52}: 01$

$$
E(\boldsymbol{x})=\frac{1}{2} \int_{\Omega_{\chi}} m\|\nabla \boldsymbol{x}\|^{2} d \boldsymbol{\chi}=\frac{1}{2} \int_{\Omega_{\chi}} m \frac{\partial x^{\alpha}}{\partial \chi^{i}} \frac{\partial x^{\alpha}}{\partial \chi^{i}} d \boldsymbol{\chi} .
$$

256 It consists in a set of decoupled quasi-linear elliptic equations mapping each coordinate. Many efficient 257 numerical methods are available to solve 


$$
\text { the }
$$

aken to avoid the degeneration of the quality of the mesh, or mesh tangling. If carefully implemented, he MMPDE TaT:03. JIN200668. ChT:08. XuN: 13, Zho: 13, ZhC: 13, PAN2015207

The design of moving mesh methods on the sphere is a relatively recent subject of research. Optimal transport method have been discussed in $\left[\begin{array}{ll}\text { WeB } \\ 58,16, M c R: 18\end{array}\right]$ using finite differences or finite-elements to solve the Monge-Ampere type nonlinear PDEs involved. The results shown in terms of mesh quality and mesh refinement are very interesting. It is however unclear whether the error reduction compensates the CPU time overhead of mesh adaptation. Based on our previous experience, here we have opted for a simpler version of mesh movement on the sphere, trying to remain as much as possible close to the simplicity of

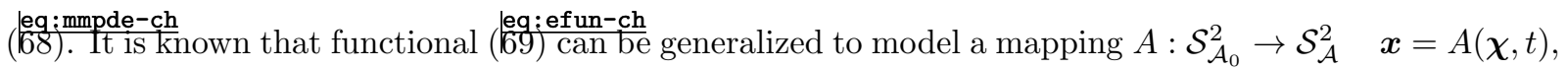
from a computational sphere with metric tensor $G_{i j}$ and local coordinates $\chi$ to a physical sphere with metric $H_{\alpha \beta}$ and local coordinates $\boldsymbol{x}$ :

$$
E(\boldsymbol{x})=\frac{1}{2} \int_{\Omega_{\chi}} m G^{i j} H_{\alpha \beta} \frac{\partial x^{\alpha}}{\partial \chi^{i}} \frac{\partial x^{\beta}}{\partial \chi^{j}} \sqrt{G} d \chi
$$

Unfortunately, the above functional is similar to the harmonic map energy of $\left[\frac{\text { DiL: } 06}{60}\right.$ where it is shown that the minimization problem associated may lack a unique solution. This is what has led several authors

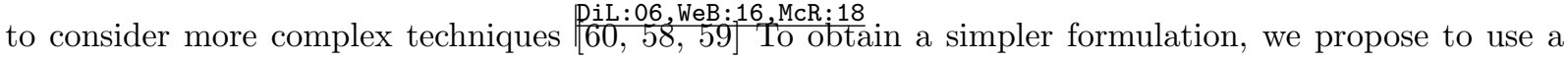
mapping form the reference sphere to the parametrization of the adapted one. So we map the sphere to a plane $A: \mathcal{S}_{\mathcal{A}_{0}} \rightarrow \mathbf{E}^{2} \quad \boldsymbol{x}=A(\boldsymbol{\chi}, t)$. This corresponds to a flat target metric is $H_{\alpha \beta}=\delta_{\alpha \beta}$ and to an energy functional:

$$
E(\boldsymbol{x})=\frac{1}{2} \int_{\Omega_{\chi}} m G^{i j} \frac{\partial x^{\alpha}}{\partial \chi^{i}} \frac{\partial x^{\alpha}}{\partial \chi^{j}} \sqrt{G} d \chi=\frac{1}{2} \int_{\Omega_{\chi}} m G^{i i} \frac{\partial x^{\alpha}}{\partial \chi^{i}} \frac{\partial x^{\alpha}}{\partial \chi^{i}} \sqrt{G} d \chi
$$

having used the fact that $G^{i j}$ is diagonal. In practice, all goes similarly to when mapping two planes.

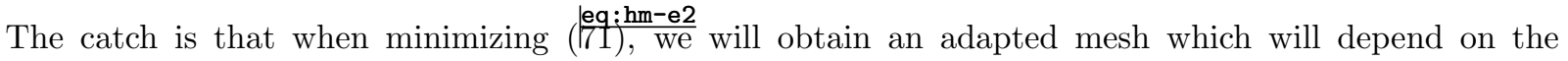
parametrization. In our opinion this is not a problem as long as the same parametrization is used for the flow equations. The MMPDE obtained in this case is

$$
\frac{1}{\sqrt{G}} \frac{\partial}{\partial \chi^{i}}\left(m \sqrt{G} G^{i i} \frac{\partial x^{\alpha}}{\partial \chi^{i}}\right)=0
$$

which, as in the Cartesian case, constitutes a decoupled system of PDEs in which the only non-linearity is associated to the monitor function $m=m(\boldsymbol{x})$ which will depend on the solution (as in the Cartesian case). We use a straightforward $P^{1}$ Finite Element method to discretize the MMPDE, and a NewtonJacobi iteration to update nodal displacements reading

$$
\boldsymbol{\delta}_{i}^{k+1}=\boldsymbol{\delta}_{i}^{k}-\left(\sum_{K \ni i} a_{i i}^{K}\right)^{-1} \sum_{K \ni i} \sum_{j \in K} a_{i j}^{K}\left(\boldsymbol{x}_{j}^{k}\right)_{K}, \quad \boldsymbol{x}_{i}^{k+1}=\boldsymbol{x}_{i}^{n}+\boldsymbol{\delta}_{i}^{k+1},
$$


subsec:mon

with $a_{i j}^{K}$ the entries of the stiffness matrix arising from a straightforward finite element approximation of $\left(\begin{array}{ll}72 \\ 2\end{array}\right)$, with the only source of nonlinearity being the monitor function $m=m(\boldsymbol{x})$. In some of the computations a standard Laplacian mesh smoothing step is also added $\frac{\mathrm{HuR}}{61,97 .}, \frac{97}{62}$.

To test the moving mesh algorithm we use a benchmark from $\frac{\text { McR: }: 18}{59}$. The monitor function is:

$$
m=1+10 \operatorname{sech}^{2}\left(5\left(\left\|x-x_{1}\right\|^{2}-\frac{\pi^{2}}{4}\right)\right)+10 \operatorname{sech}^{2}\left(5\left(\left\|x-x_{2}\right\|^{2}-\frac{\pi^{2}}{4}\right)\right)
$$

with $x_{1,2}=\left( \pm \frac{\sqrt{3}}{2}, 0,-\frac{1}{2}\right)$. The resulting mesh in shown in figure $\frac{1}{2}$ : The around the bands. The result is quite close to those presented in [59: although clearly the optimal transport method of the reference gives a smoother mesh. Somewhat nicer results are obtained when including Laplacian smoothing, as shown on the right picture on the same figure.
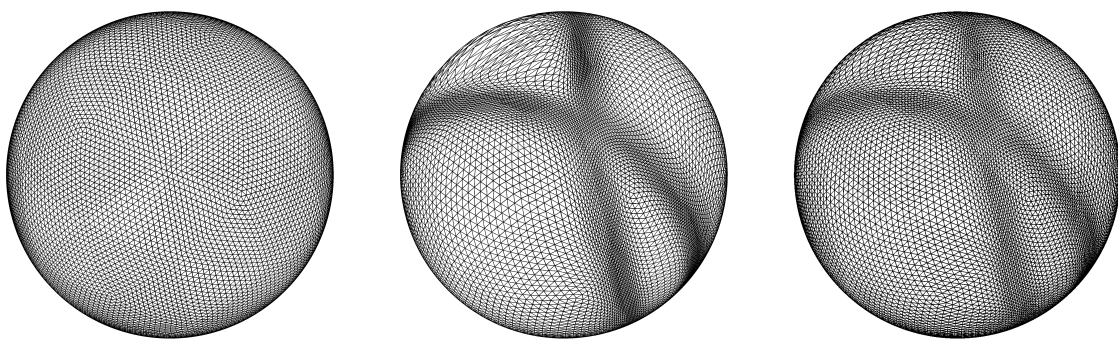

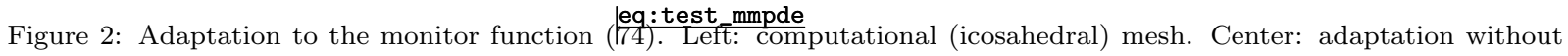
smoothing. Right: adaptation with Laplacian smoothing with smoothing coefficient $1 / 2$.

\subsection{Monitor function}

In this paper we use the arc-length type monitor function of $\frac{\text { Win: }}{63}:$ :

$$
m=\sqrt{1+\alpha_{i} f_{i *}^{2}}, \quad f_{i *}=\min \left(1, \frac{f_{i}}{\beta \max _{\boldsymbol{x} \in \Omega} f_{i}}\right)
$$

In the tests of section 8 , the physical quantities $f_{i}$ have been chosen depending on the phenomenon under

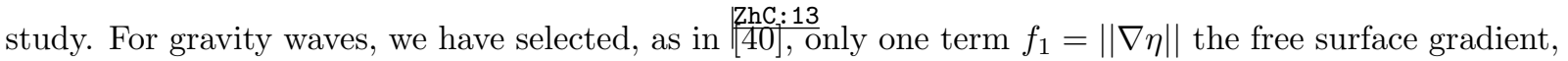
in order to detect free surface wave patterns and bore development. In presence of wet/dry transitions as in $\frac{\text { Arr: } 17}{1}$ we have added a second term $f_{2}=F(h)$ where the function $F$ is a regularized Heaviside function going from 1 to zero in vicinity of dry regions. When considering barotropic instability we have taken $f_{1}=\|\nabla \times \boldsymbol{u}\|$ and $f_{2}=\|\nabla \nabla \times \boldsymbol{u}\|$ in order to follow roll-up of the vorticity field. For all other details on our implementation of the MMPDE method please refer to Ar 1$]$ and references therein. The values of the tuning coefficients $\alpha_{i}, \beta$ are given for each test in section 8 


\subsection{Moving Mesh PDE-ALE algorithm}

For adaptive computations, the MMPDE-ALE algorithm starts with a triangulation at time $n$ and the solution $\mathrm{w}_{\mathrm{h}}^{n}$. Setting the initial conditions for the MMPDE $m_{\mathrm{h}}^{1}=m_{\mathrm{h}}^{n}$ and $\boldsymbol{x}^{1}=\boldsymbol{x}^{n}$, where $m_{\mathrm{h}}^{n}$ is the monitor function evaluated with $\mathrm{w}_{\mathrm{h}}^{n}$, we proceed as follows.

DO $k=1, k \max$

Step 1. Move the mesh according to the Newton-Jacobi iteration (69).

Step 2. Use the ALE schemes to get a remap on the new mesh as discussed e.g. in $\left[\begin{array}{l}\text { If } \\ \text { and }\end{array}\right.$ the monitor function using the new solution

ENDDO

Step 3 Let $\boldsymbol{x}^{n+1}=\boldsymbol{x}^{\mathrm{kmax}+1}$ define the new triangulation, evolve the underlying balance law in ALE framework on the half time step mesh as discussed in section $\mid \frac{|l|}{6}$ : RD

Step 5. IF $\left(t=T_{\text {final }}\right)$ EXIT ELSE GO TO Step 1.

As remarked in $\frac{\mid A r R: 17}{1]}$, the remap from one mesh to the new one here is only needed to evaluate the error function. This allows to simplify the mapping if necessary. The interested reader can refer to $\frac{\mid A r R: 17}{1}$ for a discussion.

\section{Numerical experiments}

In all computations presented hereafter we have set, unless otherwise stated, $R=6371.22 \times 10^{3} \mathrm{~m}$, $g=9.80616 \mathrm{~m}^{2} \mathrm{~s}^{-1}$ and $\Omega=7.295 \times 10^{-5} \mathrm{~s}^{-1}$. Meshes with two topologies have been tested: one generated with the unstructured mesh generator Gmsh [6uR: 64 , and more regular icosahedral grids. The typical mesh topologies are illustrated on figure $\frac{1 f i g: \text { sphere-mesh }}{3}$ Adaptation is performed with 5 iteration of the Newton-Jacobi method (1/73). Whenever additional smoothing steps are used, this is explicitly said.

\subsection{Fixed grid: zonal flows of Williamson et al.}

In this paragraph we test only the Eulerian (fixed grid) RD on some classical benchmarks taken from the test suite of $\frac{111}{65}$. 92 Grid convergence studies gave been conducted on 5 meshes generated with Gmsh and with reference sizes starting from $h_{K} R^{-1}=0.16$ and halved from one mesh to the other. The meshes thus obtained are composed of 1306, 4990, 19568, 75478, and 300426 triangles respectively.

Relative errors are evaluated following $\frac{\text { Wi1: }}{65}$ as:

$$
l_{2}=\frac{\|e\|_{L 2}}{\left\|h_{e x}\right\|_{L 2}}
$$



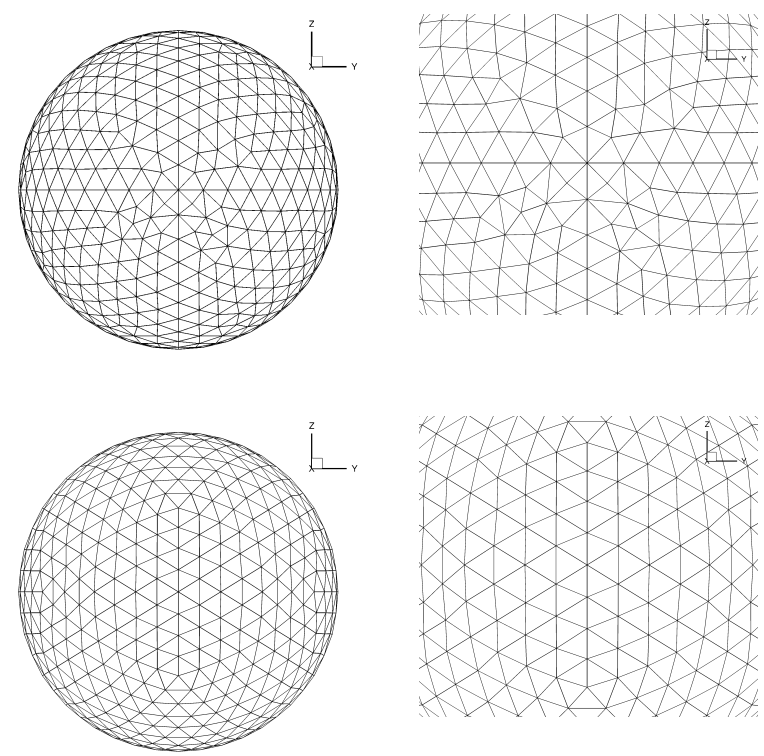

Figure 3: Computational grids on the sphere. Top row: mesh \#1 generated with gmsh. Bottom row: third level of refinement for icosahedral mesh.

with $h_{e x}$ the exact solution and

$$
\|e\|_{L 2}=\sqrt{\int_{0}^{2 \pi} \int_{-\pi / 2}^{\pi / 2}\left(h(\boldsymbol{x})-h_{e x}\right)^{2} \sqrt{G} d \boldsymbol{x}}
$$

Case \#2 is an exact steady geostrophic equilibrium allowing to measure the order of accuracy in presence of Earth rotation. The velocity ( given directly in covariant basis) and height fields are initially given by:

$$
\begin{aligned}
h(\boldsymbol{x}, 0) & =h_{0}-\frac{1}{g}\left(\Omega R u_{0}+\frac{u_{0}^{2}}{2}\right)(-\cos \lambda \cos \varphi \sin \alpha+\sin \lambda \cos \alpha)^{2} \\
u^{1}(\boldsymbol{x}, 0) & =u_{0}(\cos \alpha+\cos \varphi \tan \lambda \sin \alpha) \\
u^{2}(\boldsymbol{x}, 0) & =-u_{0} \sin \varphi \sin \alpha
\end{aligned}
$$

with $g h_{0}=2.94 \times 10^{4} \mathrm{~m}^{2} \mathrm{~s}^{-2}$ and $u_{0}=\frac{2 \pi R}{122 a y s}$. Two orientations are tested $\alpha=0$ and $\alpha=\pi / 4$. The errors obtained at day 5 are reported on table 2 below. The total error obtained on unstructured triangulations is comparable to previous results such as the Lax-Wendroff scheme on a cubed staggered grid of $\frac{R o s: 06}{3}$. The expected second order of accuracy is obtained.

As it is customary we report on figure $\frac{\text { fig }}{4}$ the error maps after 5 days of simulation on the fourth grid $\left(h_{K} R^{-1}=0.02\right)$. Particular attention should be put along the interface line (it is a discontinuity for the coordinates). Error iso-countours are very smooth for the RD scheme proposed, with seemingly no effect of the patching treatment used for the poles. For comparison we also report on figure 5 the error plot 


\begin{tabular}{|c|c|c|c|}
\hline grid \# $(\alpha=0)$ & RD & grid \# $\left(\alpha=\frac{\pi}{4}\right)$ & RD \\
\hline \hline 1 & $4.176589 \mathrm{e}-03$ & 1 & $1.656259 \mathrm{e}-02$ \\
\hline 2 & $9.286403 \mathrm{e}-04$ & 2 & $3.496469 \mathrm{e}-03$ \\
\hline 3 & $1.846086 \mathrm{e}-04$ & 3 & $9.512542 \mathrm{e}-04$ \\
\hline 4 & $4.462182 \mathrm{e}-05$ & 4 & $2.466671 \mathrm{e}-04$ \\
\hline 5 & $1.028603 \mathrm{e}-05$ & 5 & $6.292595 \mathrm{e}-05$ \\
\hline Order & 2.17 & Order & 2.00 \\
\hline
\end{tabular}

Table 1: Steady zonal flow. $l_{2}$ error.

relative to the computation on the fourth-level icosahedral mesh (81920 nodes) which has roughly the same reference size. The error $\left(\|e\|_{L 2}=4.084761 e-05\right.$ for $\alpha=0,\|e\|_{L 2}=2.634469 e-04$ for $\left.\alpha=\frac{\pi}{4}\right)$ result is very close, showing no impact of the mesh topology.
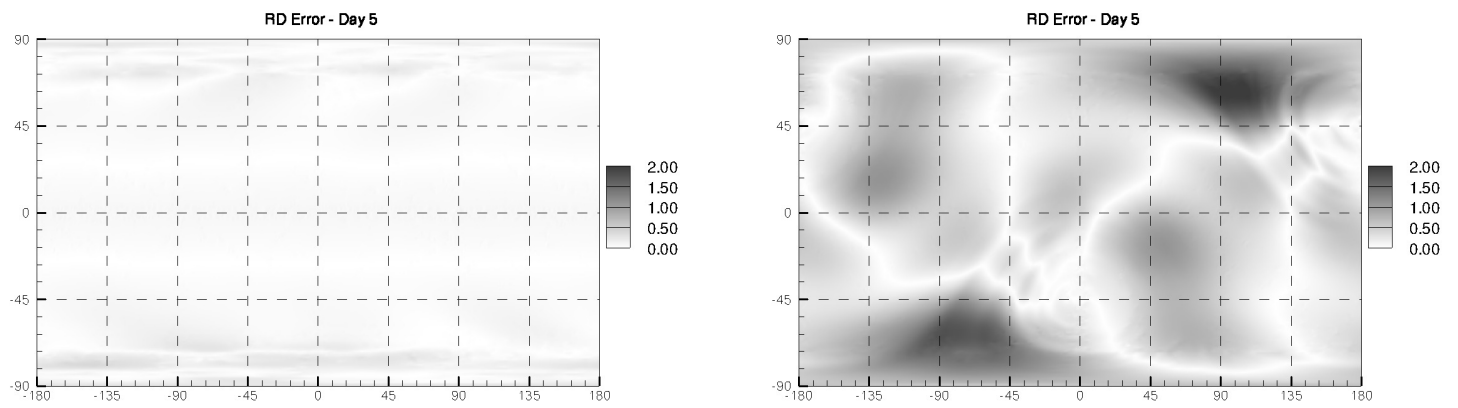

Figure 4: Steady zonal flow. Absolute value of local error on gmsh grid $h_{K} R^{-1}=0.02$. Black areas correspond to $2 m$ error, white areas to zero error. Left: $\alpha=0$. Right: $\alpha=\pi / 4$.
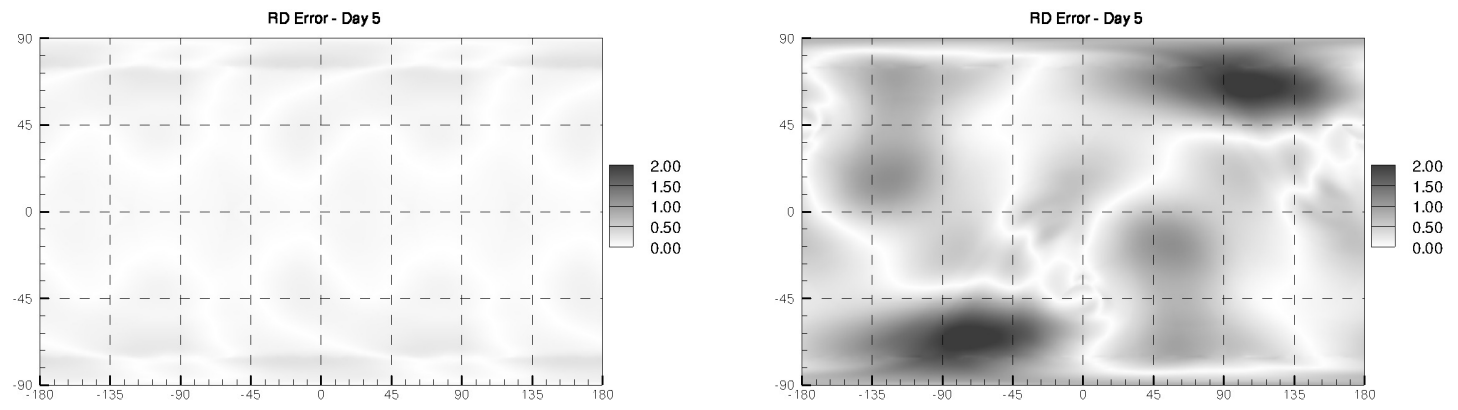

Figure 5: Steady zonal flow. Absolute value of local error on icosahedral grid (forth-level refinement) $h_{K} R^{-1} \approx 0.02$. Black areas correspond to $2 m$ error, white areas to zero error. Left: $\alpha=0$. Right: $\alpha=\pi / 4$. 
Case \#5 is a perturbation of the previous one. The initial velocity and height fields are given by (17\% with $\alpha=0, h_{0}=5960 \mathrm{~m}$ and $u_{0}=20 \mathrm{~m} / \mathrm{s}$. An isolated mountain is now added with the profile:

$$
b=b_{0}\left(1-\frac{r}{R}\right)
$$

with $b_{0}=2000 m, R=\pi / 9, r^{2}=\min \left(R^{2},\left(\varphi-\varphi_{0}\right)^{2}+\left(\lambda-\lambda_{0}\right)^{2}\right)$ and with the center of the cone in $\left(\varphi_{0}, \lambda_{0}\right)=(3 / 2 \pi, \pi / 6)$. Simulations are performed on the fourth mesh of the gmsh suite $\left(h_{K} R^{-1}=0.02\right)$.

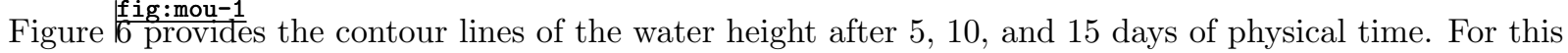
case one typically checks the conservation of total mass and energy:

$$
E=h\left(\frac{1}{2}\|\boldsymbol{u}\|^{2}+g \frac{h}{2}+g b\right)
$$

Note that mass error is only related to the change of the coordinate system across the edges that delimit the polar cap. Regarding energy, none of the schemes proposed have provable energy conservation properties, which makes the definition of the actual discrete evaluation of the energy somewhat arbitrary. In practice, here the energy is computed directly from nodal quantities, and then integrated using a linear

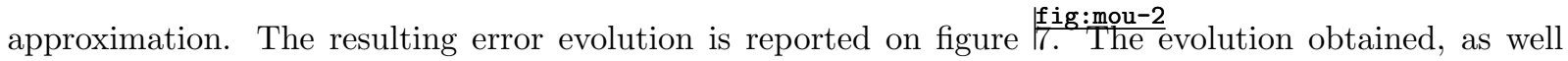
as errors obtained, for the mass and energy are comparable to or better than those typically reported in literature for computations on structured grids, and with the high resolution results by the German Weather Service (DWD) http://icon.enes.org/swm/stswm/node5.html.
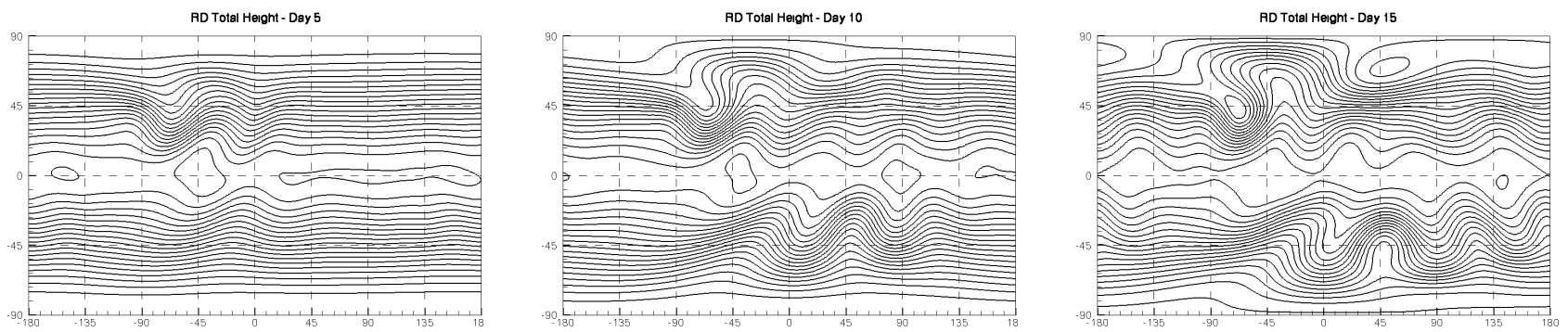

Figure 6: Zonal flow over an isolated mountain. Snapshot of the numerical solution for fixed grid simulation on gmsh mesh $h_{K} R^{-1}=0.02$ at day 5,10 and 15 . Contour levels are from $5050 \mathrm{~m}$ to $5950 \mathrm{~m}$ in intervals of $50 \mathrm{~m}$.

\subsection{Advection of cosine bell}

To assess the numerical accuracy of our algorithm for unsteady problems and in presence of mesh movement, we revisit test \#1 of the Williamson suite $\frac{\frac{111}{65}: 92}{6 .}$ For this test, the advective component is tested in isolation: only the mass equation is a prognostic equation while the wind (always expressed in 

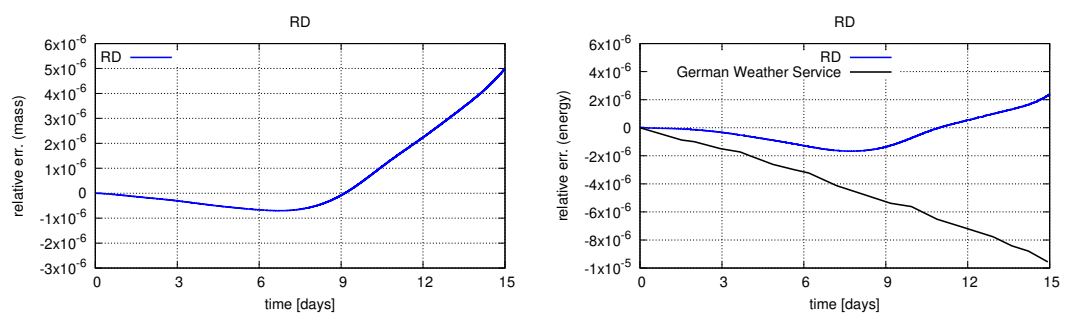

Figure 7: Zonal flow over an isolated mountain. Relative conservation error for mass (left) and energy (right) on gmsh mesh $h_{K} R^{-1}=0.02$.

covariant components) is constant and given by

$$
\begin{aligned}
& u^{1}(\boldsymbol{x}, 0)=u_{0}(\cos \alpha+\cos \varphi \tan \lambda \sin \alpha) \\
& u^{2}(\boldsymbol{x}, 0)=-u_{0} \sin \varphi \sin \alpha
\end{aligned}
$$

We compute the transport of a cosine bell given by:

$$
h(\boldsymbol{x}, 0)= \begin{cases}\frac{h_{0}}{2}(1+\cos (3 r))+h_{0} & \text { if } r<\pi / 3 \\ h_{0} & \text { otherwise }\end{cases}
$$

where $h_{0}=1000, u_{0}=\frac{2 \pi R}{12 \text { days }}$, and $r$ is the great circle distance from the center of the bell $\left(\varphi_{C}, \lambda_{C}\right)$ :

$$
r=R \arccos \left(\sin \lambda_{C} \sin \lambda+\cos \lambda_{C} \cos \lambda \cos \left(\varphi-\varphi_{C}\right)\right)
$$

We have tested two different wind directions $\alpha=0$, and $\alpha=\pi / 2$ for which the bell is transported through the north and south poles, crossing four times a polar cap interface. To test the accuracy of the ALE formulations we have added an independent unsteady motion of the mesh nodes according to the transformation:

$$
\begin{aligned}
& \varphi(t)=\Phi+0.5 \cos (\Phi) \cos (\Lambda) \sin \left(\frac{4 \pi t}{T}\right) \\
& \lambda(t)=\Lambda+0.5 \cos (\Phi) \cos (\Lambda) \sin \left(\frac{4 \pi t}{T}\right)
\end{aligned}
$$

with $\Phi, \Lambda$ the computational or reference coordinates. The typical deformations obtained can be seen on figure $\frac{f i g: c b-m e s h}{8,}$ This motion will act as a background perturbation to the cosine bell advection.

The mesh convergence for fixed and moving meshes is reported on figure 90 The expected second order of accuracy is recovered. Moreover, we see roughly the same level of error for the two configurations (zonal and meridional advection). This suggests that the polar patching does not affect significantly the error constant, nor the slope. We also observe that the unsteady mesh distortion does not spoil neither the order of accuracy nor the error level. This validates the ALE formulation, at least in terms of accuracy 

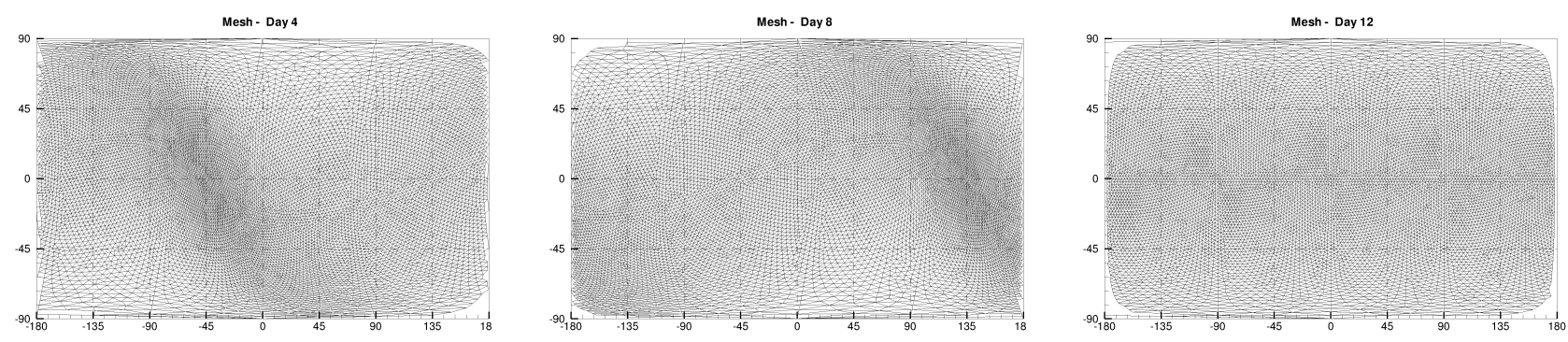

Figure 8: Advection of cosine bell. Transformed meshes number 2 at three different instants. At day 12 (final time) the transformed mesh coincides with the computational mesh.
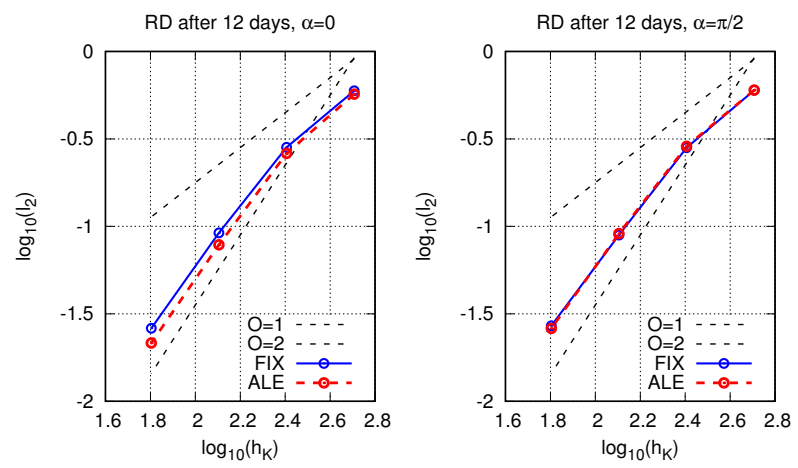

Figure 9: Advection of cosine bell: grid convergence. Left: $\alpha=0$. Right: $\alpha=\pi / 2$ fig:cb-conv

\subsection{Circular hump}

This is a test proposed in $\frac{\text { Ros:04 }}{2}$. The initial condition is a circular depth disturbance at the equator:

$$
h(\boldsymbol{x}, 0)=\left\{\begin{array}{l}
2 \text { if } \arccos \left(\cos \left(x^{1}\right) \cos \left(x^{2}\right)\right) \leq 0.2 \\
0.2 \quad \text { otherwise }
\end{array}, \quad \boldsymbol{u}(\boldsymbol{x}, 0)=0\right.
$$

This initial condition is symmetric about the point $\left(x^{1}, x^{2}\right)=\left(0^{\circ}, 0^{\circ}\right)$, and should remain symmetric in absence of rotation. We run computations on two half sphere meshes generated with gmsh: a coarse one with 7122 points and 14034 elements; a fine one with 39699 and 78900 elements. Adaptive computations on the coarse grid are performed using the MMPDE of section 1 sec:mmpde and the ALE formulation. We report the solution contours obtained with the RD scheme on figure $\frac{f i g}{10}$ : ch-iso We can see on all meshes good shock capturing and good symmetry in all radial directions. We can observe that the mesh adaptation itself also preserves a good symmetry of the grid, and allows an extremely sharp resolution of the inner and outer shocks. 

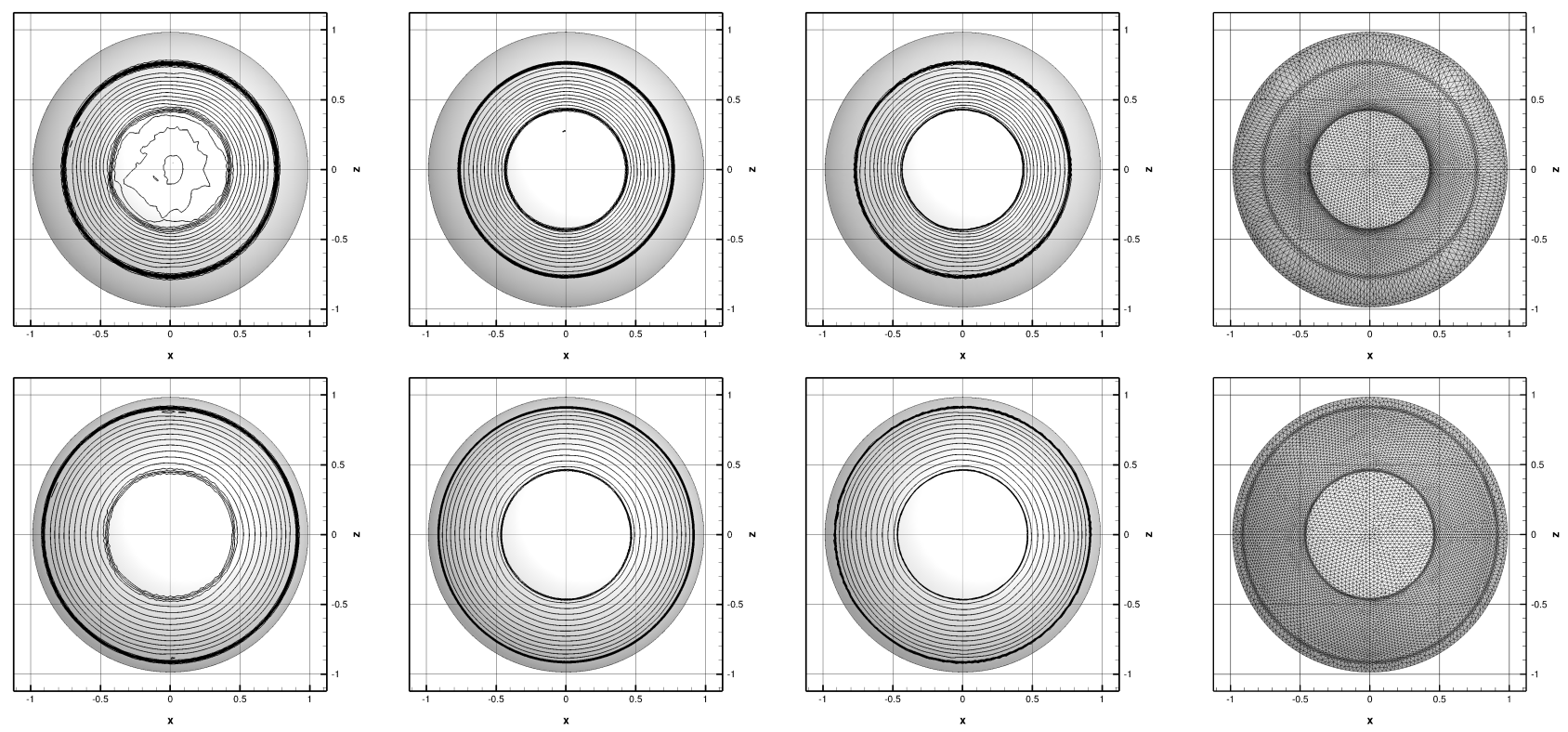

Figure 10: Circular hump on a sphere: depth iso-contours (RD solutions, 20 levels between 0 and 0.55 ) at times $t=0.6$ (top) and $t=0.9$ (bottom). From left to right: fixed coarse mesh solution, fixed fine mesh solution, ALE adaptive mesh solution, adaptive mesh.

To further evaluate the results on figure:ch-cut

To further evaluate the results on figure 11 we plot the line cuts of the fluid depth along the equator. In the same figure our RD implementation is compared against the numerical solution of $\frac{\text { Ros: } 04}{2}$, obtained with high-resolution FV (Lax-Wendroff flux with MC limiter) on a Cartesian grid composed of 34680 points. We can see the sharp capturing of the discontinuities on all grids, and the improvement brought by the MMPDE adaptation on the coarse one. In these plots the adaptive mesh solution is indistinguishable from the fine mesh one.

Finally on table $\frac{t a b: c h-c p u}{2}$ we report the CPU times for all computations. We compute the overall time and also isolate the time associated to the MMPDE part (both mesh equation, and solution projections). We can see that the moving adaptive mesh computations are twice less expensive than the fine mesh ones, and also that even with our simplified formulation adaptation counts for non-negligible component of the simulation, mode than half for the RD scheme.

We repeat the same test adding the effects of the rotation. We set the dimensionless rotation rate to $\Omega=5$. In figure $\frac{\text { fig }}{12}$ : chc-iso we report snapshots of the depth contour lines on fixed (coarse and fine) and adaptive meshes. As in the previous case, we see the higher resolution obtained with the adaptive moving mesh and the ALE formulation. This time mesh nodes are clustered both around the shock waves and also in correspondence of smoother features where high gradients appear. On table 3 we report the CPU times. 

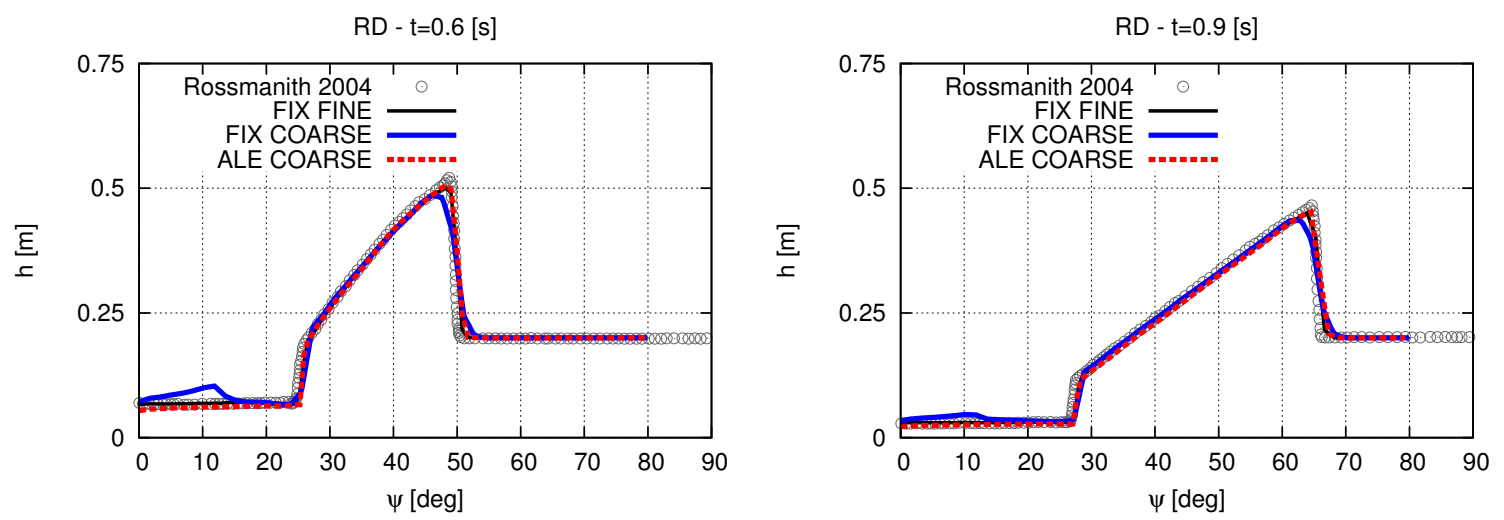

Figure 11: Circular hump on a sphere. Solution along the equator.

\begin{tabular}{|c|c|c|}
\hline ALG & Mesh (Nodes) & $\mathrm{RD}[\mathrm{s}]$ (\%MMPDE) \\
\hline \hline FIX-COARSE & 7122 & 46.89 \\
\hline FIX-FINE & 39699 & 674.66 \\
\hline ADAPT-ALE & 7122 & $368.57(51.5 \%)$ \\
\hline
\end{tabular}

Table 2: Circular hump on a non rotating sphere. CPU times.

\subsection{Barotropic instability}

This test, contained in $\frac{6 a 1}{66}: 04$, consists of a geostrophically balanced mid-latitude jet, to which a small perturbation is added to initiate an instability. Here we test the RD scheme on a fine mesh with reference size of $h_{K} R^{-1}=0.01$, and compare with adaptive simulations on coarse grids with $h_{K} R^{-1} \approx 0.02$. The fine mesh has roughly 300k triangles, while the coarse ones have approximately $80 \mathrm{k}$ triangles. For the adaptive simulations we compare results on the icosphere as well as on a gmsh mesh. In particular, adaptation has been performed using in the monitor function relative vorticity and its gradient through the

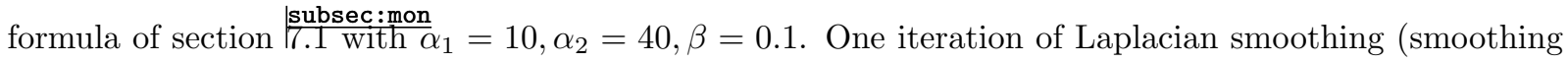
coefficient 0.25 ) is used.

\begin{tabular}{|c|c|c|}
\hline ALG & Mesh (Nodes) & RD $[\mathrm{s}]$ (\%MMPDE) \\
\hline \hline FIX-COARSE & 7122 & 81.39 \\
\hline FIX-FINE & 39699 & 1056.39 \\
\hline ADAPT-ALE & 7122 & $638.22(50.2 \%)$ \\
\hline
\end{tabular}

Table 3: Circular hump on a rotating sphere. CPU times. 

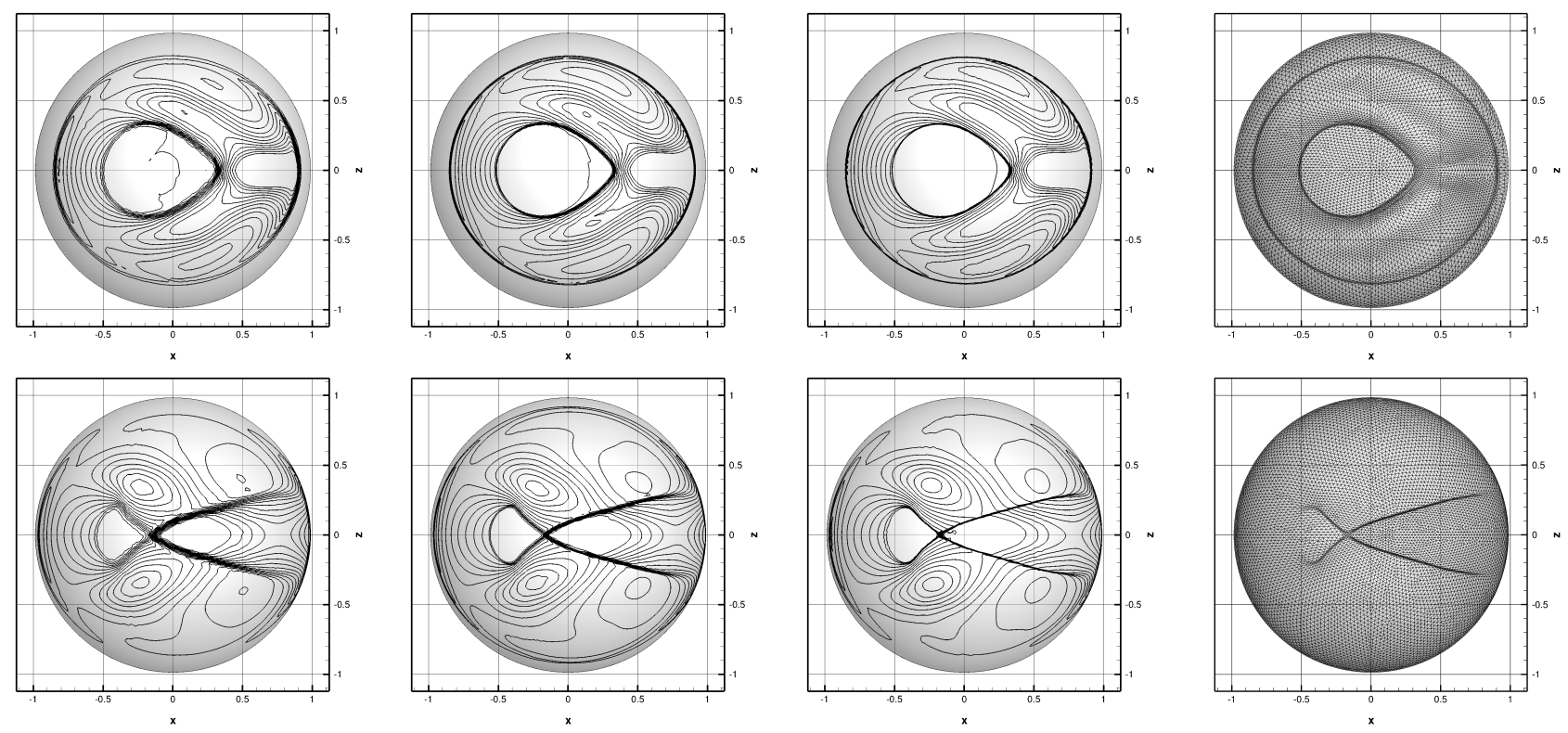

Figure 12: Circular hump on a rotating sphere: depth iso-contours (RD solutions, 20 levels between 0 and 0.55 ) at times $t=0.8$ (top) and $t=1.2$ (bottom). From left to right: fixed coarse mesh solution, fixed fine mesh solution, ALE adaptive mesh solution, adaptive mesh.
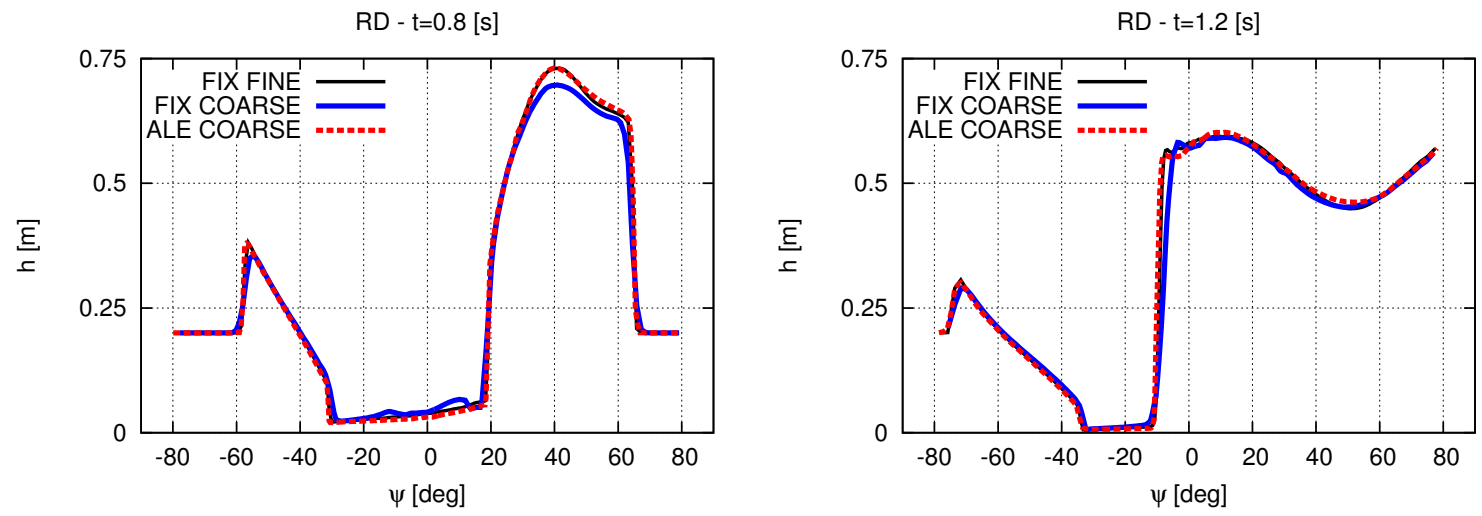

Figure 13: Circular hump on a rotating sphere. Solution along the equator. 


\begin{tabular}{|c|c|c|}
\hline ALG & Mesh (Nodes) & RD [s] (\%MMPDE) \\
\hline \hline FIX-FINE & gmsh 150215 & 246629.4 \\
\hline ADAPT-ALE & gmsh 41681 & $116126(53.3 \%)$ \\
\hline ADAPT-ALE & icosahedral 40962 & $86642.7(52.8 \%)$ \\
\hline
\end{tabular}

Table 4: Barotropic instability. CPU times.

In figure $\frac{\text { fiog: } 10 \text { al }}{14}$ we show the vorticity field corresponding to a physical time of 6 days. The top figure reports the field obtained with the fine mesh, which is very similar to the results typically reported in

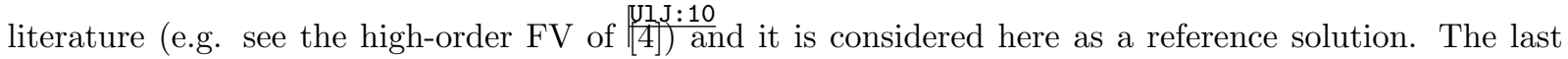
row on the bottom show the same fixed mesh computation on the coarse grid. We can clearly see that the onset of the instability as well as its evolution are wrongly reproduced on this mesh level. Note that the result is the same independently on the mesh topology, the icosahedral mesh providing very similar results to the one generated with gmsh.

Second and third rows report the adaptive simulations on the coarse meshes. We can clearly see that the adaptive mesh movement allows to correctly reproduce the global instability process, with a slight smoothing of last part of the instability $\left(\varphi \leq \sim-180^{\circ}\right)$ for the gmsh mesh, while the result on the coarse adaptive icosahedral is almost identical to the fine mesh one. 3D visualization of the vorticity fields on the adaptive meshes and the corresponding meshes obtained are reported on figure $\frac{\text { f ig }}{15}$ : gal1 showing a nice capturing of the instability. Finally, we report the computational times on table 渵 cost more then one third of the fine mesh simulation. This is an encouraging result confirming the potential of the schemes and of the mesh adaptation technique proposed here.

\subsection{Adaptive simulation of the Tohoku 2011 tsunami}

As a last application we consider the simulation of the 2011 Tohoku-Honsu tsunami with the RD scheme and the MMPDE-ALE approach proposed. The computational domain is a relatively large chunk of the Pacific ocean $(650[\mathrm{~km}] \times 650[\mathrm{~km}])$, on which an initial waveform computed in $\frac{p \mathrm{rgm}: 17}{67}$ with the approach of $\frac{\mathrm{SaF}}{62}$ : 13 is imposed. To asses the potential of the MMPDE-ALE approach on a multiscale problem, we consider the initial propagation from the source to the coast, and the runup and flooding of the complex bathymetry of the coast. Both topographic data and initial wave elevation and currents are provided by BRGM within the research program TANDEM (see http://www-tandem.cea.fr). A sketch of the domain and of the initial solution is reported on figure $1 \frac{f i g}{16}$. toho Friction is based on a constant Manning coefficient of $n=0.03125\left[\mathrm{sm}^{-1 / 3}\right]\left[\frac{\mathrm{GaL}: \mathrm{La}}{\mathrm{6g}}\right]$. 

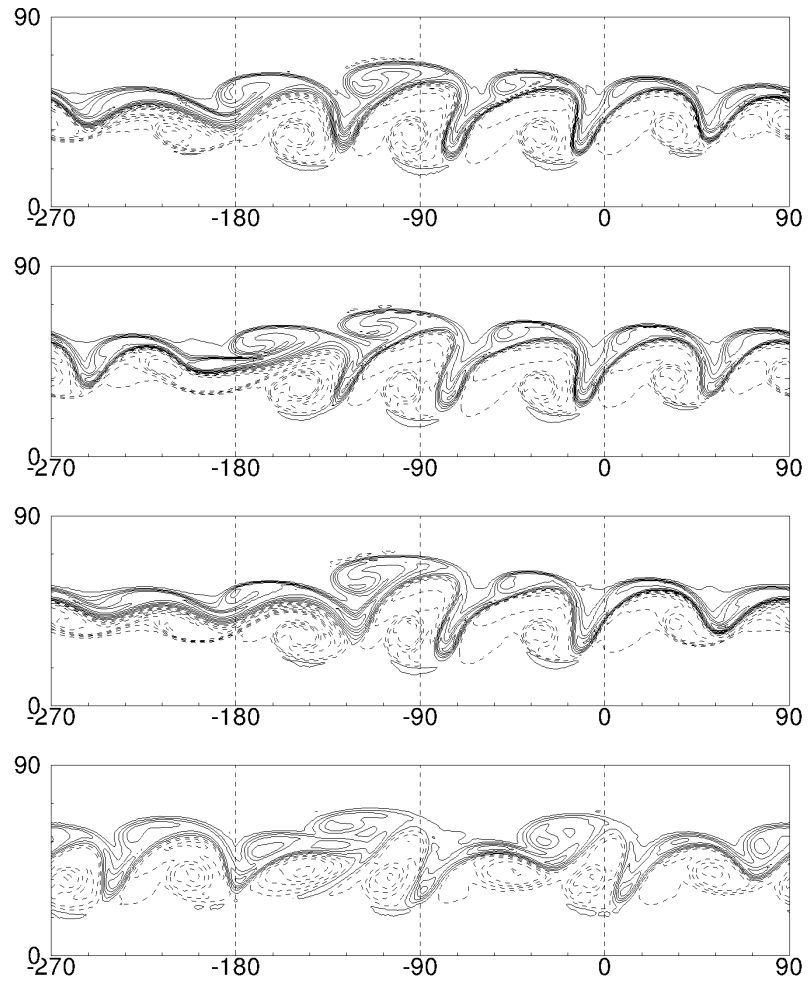

Figure 14: Barotropic instability. Vorticity field at day 6. Contour lines are from $-1.1 e-4 s^{-1}$ to $-1.5 e-4 s^{-1}$ in intervals of $2 e-5 s^{-1}$. Top: fixed fine mesh. Second and third rows: adaptive (respectively icosahedral and gmsh) coarse mesh. Bottom: fixed coarse. 

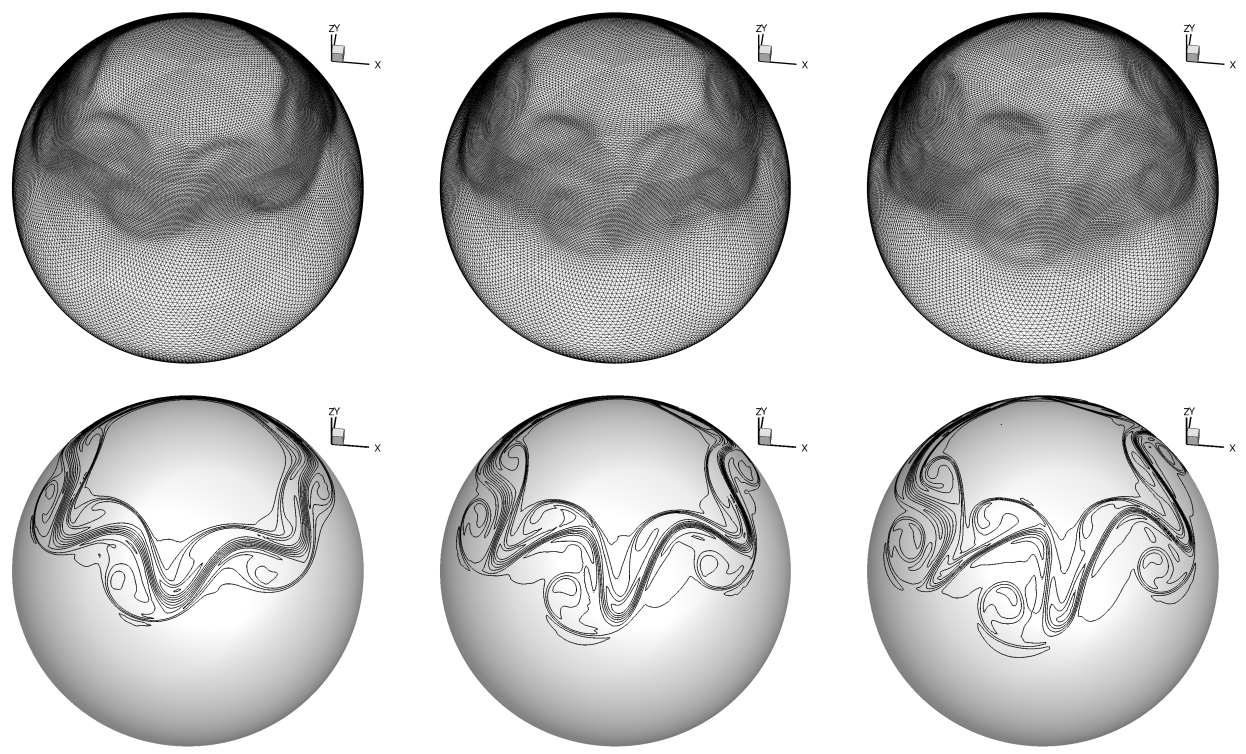

Figure 15: Barotropic instability. Relative vorticity field with corresponding adapted mesh at day 5, 5.5 and 6.
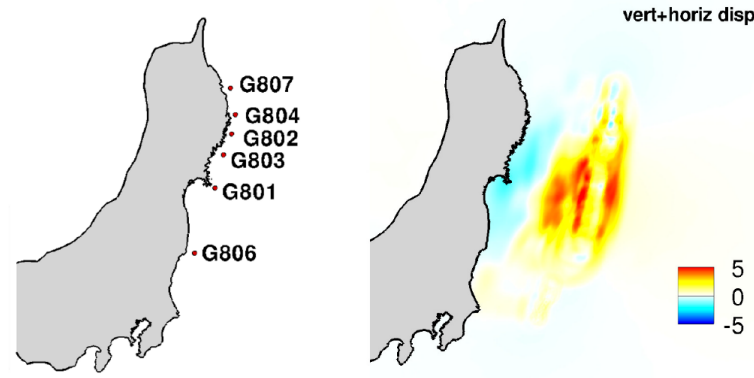

Figure 16: Tohoku-Honsu tsunami: domain with position of the GPS probes (left) and initial wave height (right). Colour legend in meters.

A reference computation has been run on a fine unstructured mesh strongly refined in proximity of the coast, where the mesh size is reduced from $5 \mathrm{~km}$ used offshore to $120[\mathrm{~m}]$. The coarse mesh used for the MMPDE-ALE method has local mesh size respectively of $360[\mathrm{~m}]$ in the proximity of the coast, and an offshore size of $15 \mathrm{~km}$. This allows to cut by a half the number of mesh elements resulting in a mesh composed by 364864 nodes and 728874 elements. Mesh adaptation is performed as discussed in section | 7 w.1 with tuning parameters $\alpha_{1}=20, \alpha_{2}=60$ and $\beta=0.1$.

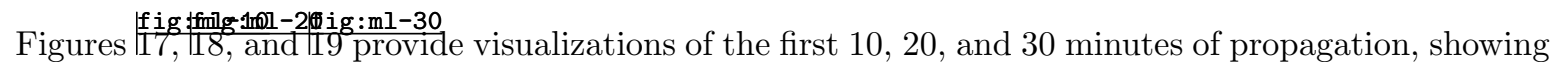
water height and the adaptive meshes obtained in vicinity of the Japanese coast. We can see how the 

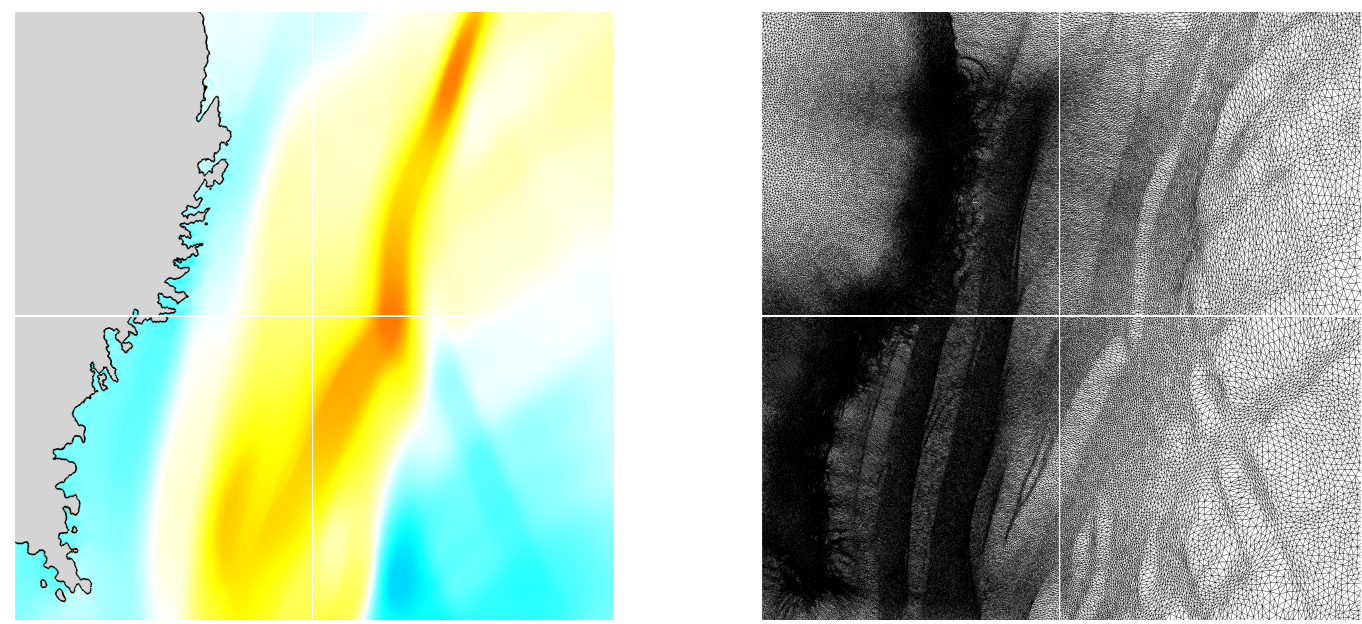

Figure 17: Tohoku tsunami. Snapshot of the free surface level (close up of the Iwate and north of the Miyagi prefectures, same colour legend of previous figures) at $t=0: 10^{\prime}$ (time origin is 11 March 2011 14:51:18 LT).

fig : ml-10
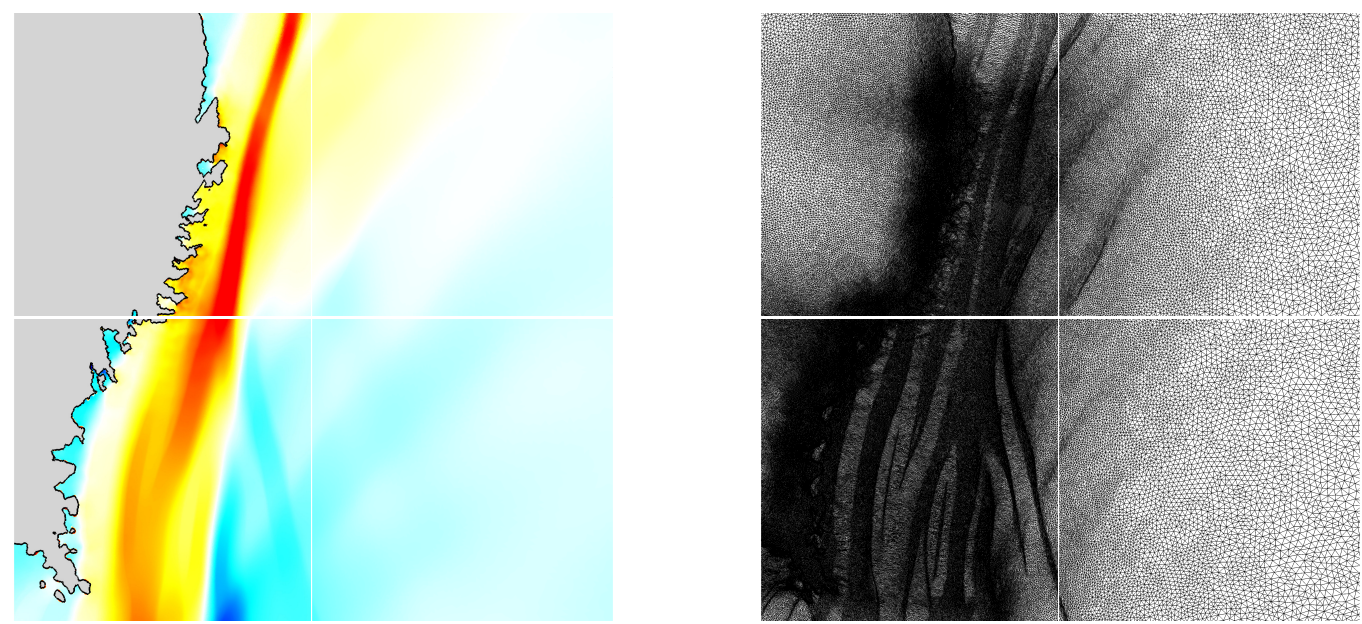

Figure 18: Tohoku tsunami. Snapshot of moving mesh (close up of the Iwate and north of the Miyagi prefectures, same colour legend of previous figures) at $t=0: 20^{\prime}$ (time origin is 11 March 2011 14:51:18 LT). 

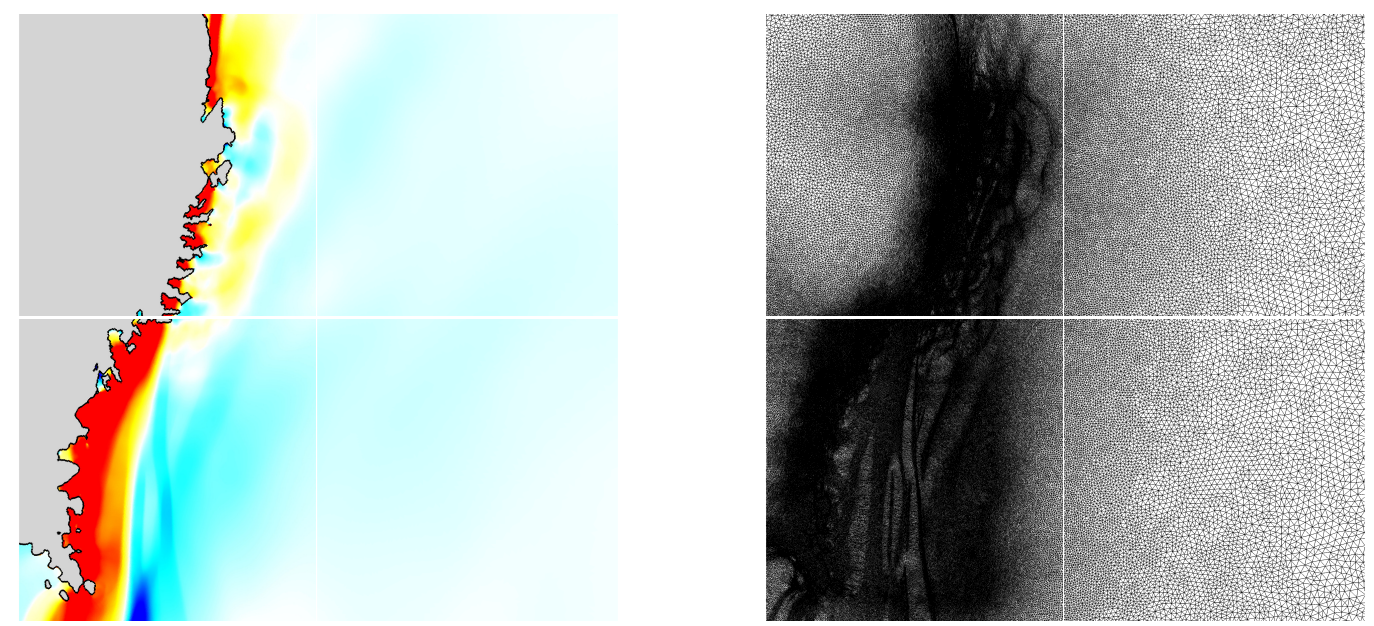

Figure 19: Tohoku tsunami. Snapshot of the free surface level (close up of the Iwate and north of the Miyagi prefectures, same colour legend of previous figures) at $t=0: 30$ ' (time origin is 11 March 2011 14:51:18 LT).

mesh adapts to the complex tsunami wave pattern. This becomes visible especially when the wave shoals and the mesh points gather in correspondence of the largest gradients of the incoming waves train. To further evaluate the quality of the adaptive computations we compare on figure $\frac{10 \mathrm{~g}: \mathrm{ssh}}{20}$ the time series of the water elevation in the GPS gauges. Both the fine mesh and the adaptive ones agree quite well with the GPS observations, with an accurate computation of the arrival time and of the peak of the leading wave. The complex interaction between incoming and trapped waves that follows is also well reproduced, see others published results $\frac{\text { Che: } 14, \text { LoK: } 12, \text { ShS: } 12}{71}, \frac{12}{72}$. We remark that the adaptive computations allow to reproduce the fine mesh results with half of the mesh elements.

We now consider the approximation of the flooding of the Japan coastal prefectures. We focus on three bays in the south of the Iwate prefecture: they are the Kesennuma, Hirota and Ofunato bay, as they appear in figure $\frac{\mid f i g: f l o o-1}{21}$ from south to north. The figure shows on the left the fine mesh with the initial coastline superimposed, a visualization of the initial coastline in the middle and the position of the inundation front after 40 minutes from the beginning of the event. The figures give an idea of the geometrical complexity of the front line, with inundation scales of the order of magnitude of 10 meters, which can be compared with the propagation scales of several hundreds of kilometers.

The handling of this complexity with the MMPDE is represented on figure $\frac{\text { fig }}{22}$ :floo-2 showing the meshes adapted to the initial coastline and to the coastline after 40 minutes. We can see here the limitations of the moving mesh approach which is stretched here to its maximum. For some of the features, as for example the thinner lands close to the Osimaseto Strait and those around Hirota bay, clearly the density 

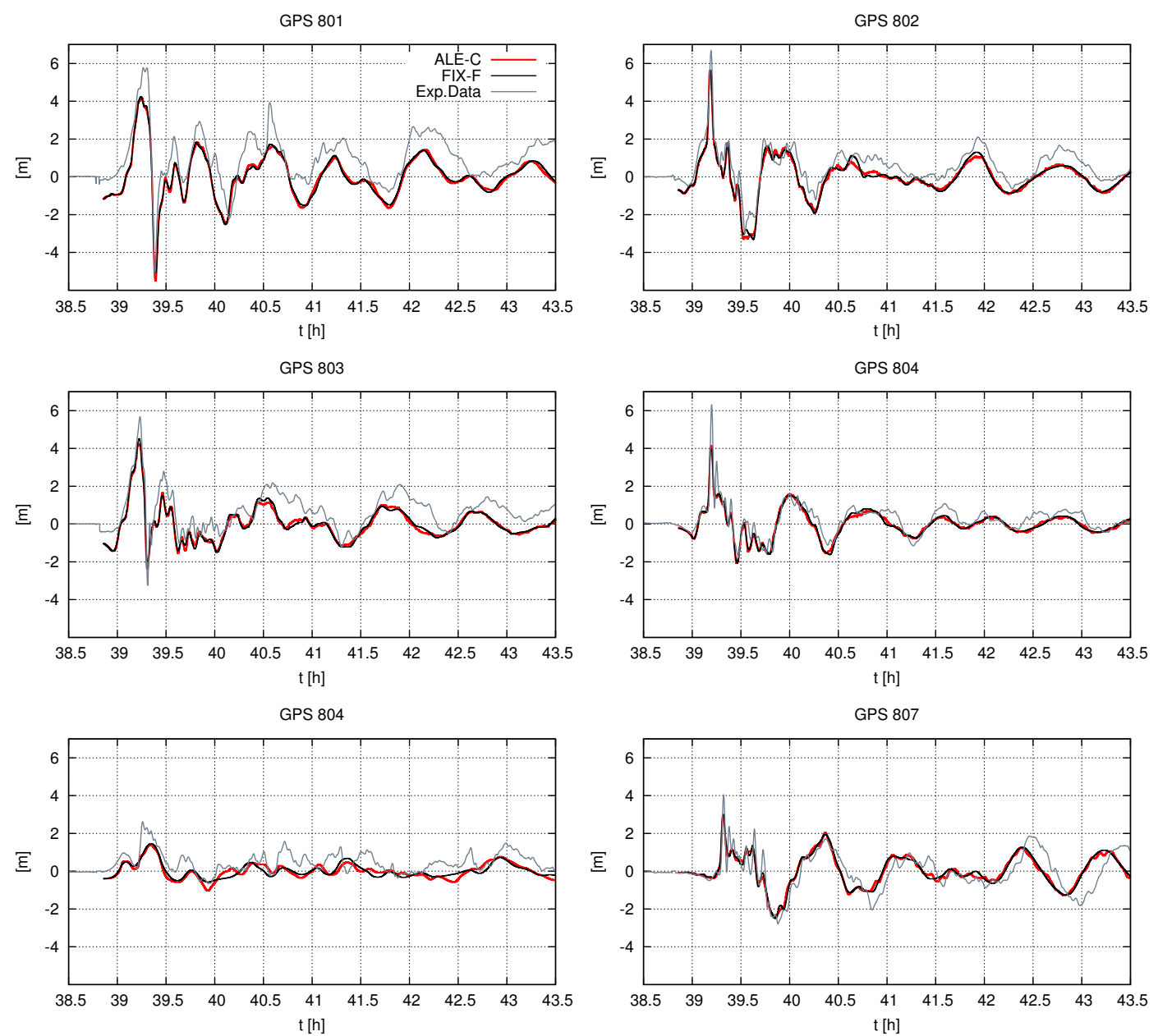

Figure 20: Simulated sea level displacement recorded at GPS buoys with fixed embedded mesh and moving embedded mesh. fig: ssh 

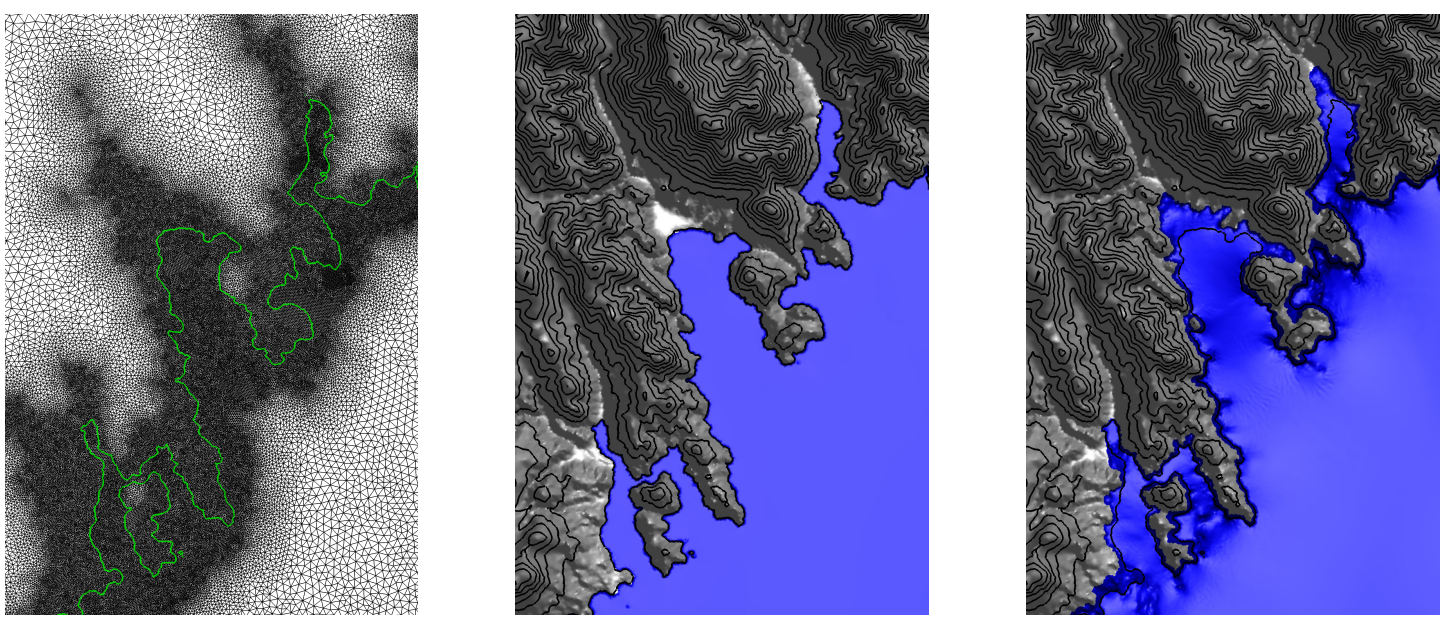

Figure 21: Tohoku tsunami. Flooding of the Iwate prefecture computed with RD scheme on fine fixed grid mesh. Left: zoom of the mesh, Right: snapshot of the inundated areas at at $t=0: 00$ ' and $t=0: 40$ ' (time origin is 11 March 2011 14:51:18 LT).

of mesh nodes is not enough to allow a capturing of all the interfaces present. This results in parts of the emerged coast to be represented essentially with one stretched element. This is especially true for the mesh at 40 minutes (right picture), and has of course an impact on the approximation of the moving wet dry front in these areas. From the same picture we can also already see that the mesh follows well the inundation front obtained on the fine mesh, and visible on the rightmost picture on figure $\mid \frac{f i g: f l o o-1}{21}$
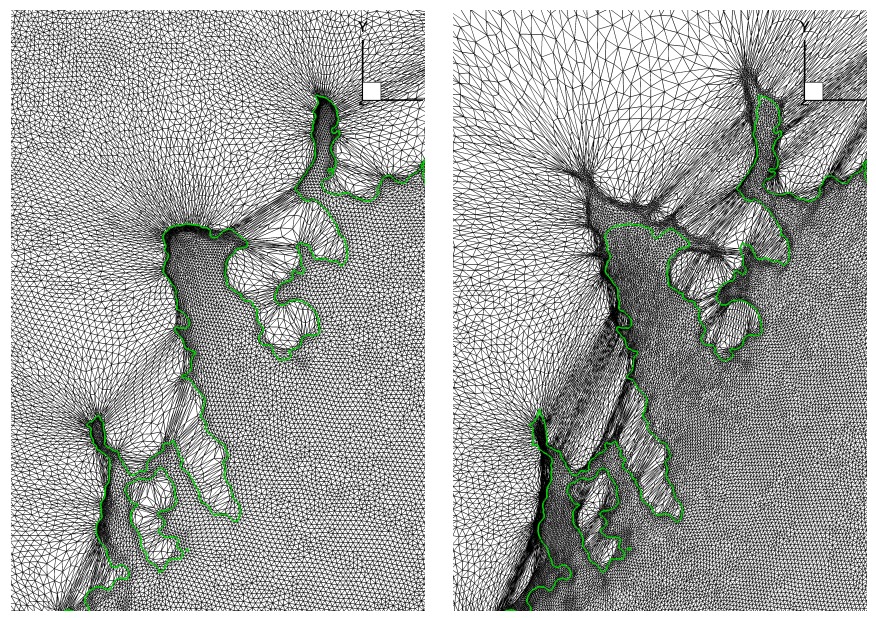

Figure 22: Tohoku tsunami. Flooding of the Iwate prefecture computed with RD scheme. Snapshot of the mesh at $t=0: 00$ ' and $t=0: 40$ ' (time origin is 11 March 2011 14:51:18 LT).

To go further in the comparison, we consider the runup plot in the same area. On figure $\frac{\text { fig }}{23}$ :floo-3 

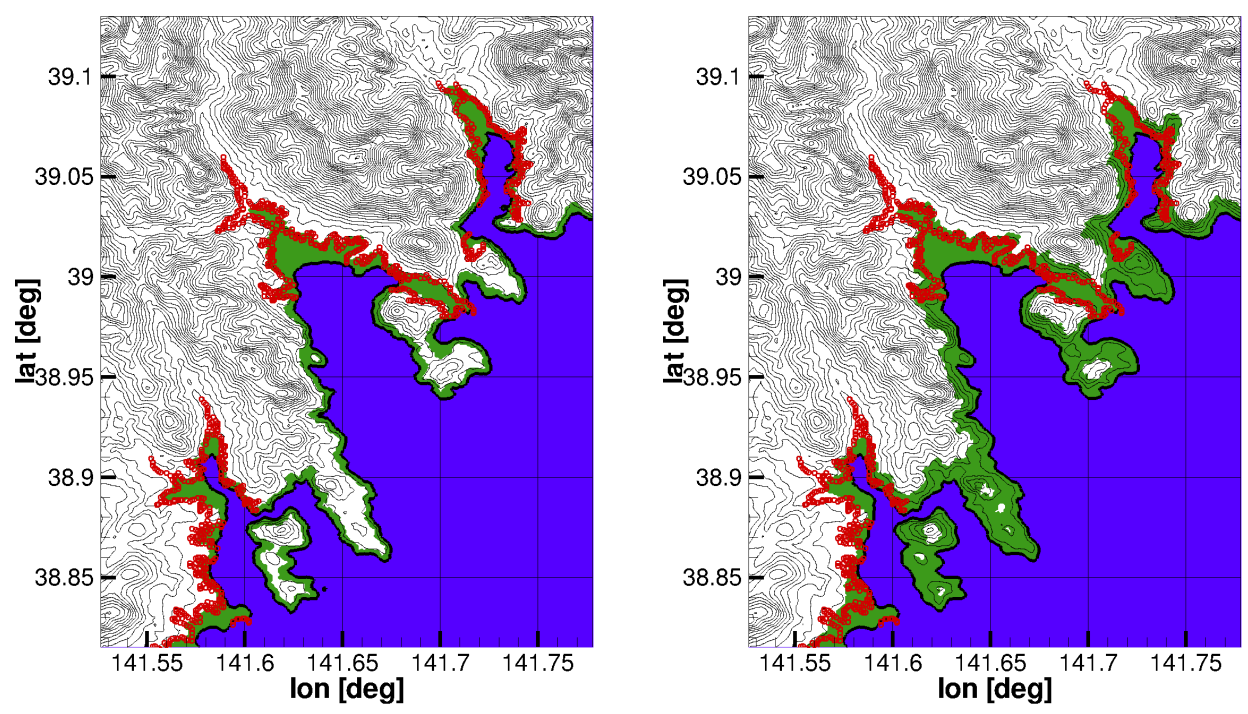

Figure 23: Tohoku tsunami. Green zones are flooded areas in Iwate prefecture. Left: fix coarse simulation. Middle: fix fine simulation. Right: adaptive ALE simulation on coarse mesh. Red dots denotes surveyed points, from $\frac{\text { Che: } 14}{70}$.

the runup obtained on the fine mesh (left picture), and with the adaptive computation (right picture). In both pictures the flooded computational areas are represented in green, while red symbols denote the surveyed inundation in the real event. The fixed fine mesh computation gives a good prediction of the runup process, with exception of some fine detail as the inundation of two narrow channels at the end of Hirota bay, which remain dry throughout the simulation.

The result of the MMPDE-ALE scheme is acceptable as it does cover most of the surveyed inundation areas. However, there is no improvement w.r.t. the fine mesh, and we clearly see the main limitation of the approach: the effect of the mesh stretching introduced in the dry region close to the wet/dry areas (cf. right picture on figure $\frac{f i g}{22}$ : $f 100-2$ geographically is unrealistically large. To cope with this phenomenon one could either look for a recipe to a-priori tune the density of mesh nodes, so that all features may be resolved. One may also try to move more points in the dry region to improve the resolution, however for such multi scale problems, a more realistic path is to combine the MMPDE with a re-meshing step. We think the result obtained here is a good example of the how much MMPDE can be stretched for this type of problems. 


\section{Conclusions and outlook}

We have discussed approximations of the rotating shallow water equations on the sphere using fixed and moving meshes. The main principles of the ALE form of the equations have been derived, and the relations between the GCL and well balancedness have been shown. A numerical approximation based on residual distribution has been proposed, with a numerical closure allowing to combine DGCL and Cproperty. A simplified elliptic PDE for mesh adaptation has been proposed and tested as a basis for a time dependent MMPDE-ALE method. The numerical results have shown that on fixed unstructured meshes the RD scheme proposed achieves accuracy close to te one usually presented in literature on structured grids. For flows with discontinuities and unstable fronts the MMPDE-ALE adaptation allows to enhance this accuracy, allowing in principle a considerable reduction in CPU time. For the cases considered here this reduction goes from $30 \%$ to $50 \%$. We have also shown that for genuinely multi-scale problems, as the Tohoku-Honsu tsunami computation of section 8.5 this technique reaches its limitations and a re-meshing phase is clearly necessary.

This work is a first step toward several generalizations which will involve both higher order approximations based on stabilized continuous polynomial approximations, an ad hoc combination of mesh movement and local remeshing, and possibly improved treatments of the pole problem, following e.g. the finite element mixed 3D/2D approximation proposed in Bern:09

\section{Acknowledgments}

Work partially funded by the TANDEM contract, reference ANR-11-RSNR-0023-01 of the French Programme Investissements d'Avenir. Some of the computations presented in this paper were carried out using the PlaFRIM experimental testbed, supported by Inria, CNRS (LABRI and IMB), Université de Bordeaux, Bordeaux INP and Conseil Régional d'Aquitaine. We are grateful to our colleagues from BRGM Orléans (and in particular R. Pedreros) for useful discussions and for the data used in the Tohoku simulations.

\section{Appendix A. Notation and concepts from differential geometry}

This appendix recalls some basic concepts from differential geometry, and used in the main body of the paper. We adopt a spacetime formalism which is better suited to time-deforming frame of reference. In particular, we work with spacetime coordinates $\left\{x^{0}, x^{1}, x^{2}\right\}$, where the first is understood to be time 
483

$x^{0}=t$, while $\left\{x^{j}\right\}_{j=1,2}$ are purely spatial ones. In all the formulas that will follow, standard summation convention is implied, unless otherwise stated.

\section{Appendix A.1. Spherical transformation}

We consider a steady transformation from the set of Cartesian coordinates $\boldsymbol{r}=\left\{r^{1}, r^{2}, r^{3}\right\}$ to a set of curvilinear coordinates $\boldsymbol{x}=\left\{x^{1}, x^{2}\right\}$. The curvilinear coordinates describe a sphere embedded in $\mathbb{R}^{3}$ with covariant vector basis which are tangent to the sphere $\left\{\boldsymbol{g}_{1}, \boldsymbol{g}_{2}\right\}$. The transformation reads:

$$
\boldsymbol{r}=F(\boldsymbol{x})
$$

We can define the Jacobian matrix of this mapping, and of the inverse mapping, as

$$
\boldsymbol{J}_{F}=\left[\frac{\partial r^{i}}{\partial x^{j}}\right], \quad \boldsymbol{J}_{F}^{-1}=\left[\frac{\partial x^{i}}{\partial r^{j}}\right]
$$

An important role is played by the metric tensor $\boldsymbol{G}$, which is a symmetric tensor that allows to define a measure of length on the sphere $d s=G_{i j} d x^{i} d x^{j}$, with

$$
G_{i j}=\boldsymbol{g}_{i} \cdot \boldsymbol{g}_{j}
$$

The metric tensor can also be evaluated directly from the mapping Jacobian using the relation

$$
\boldsymbol{G}=\boldsymbol{J}_{F}^{T} \boldsymbol{J}_{F}
$$

eq:G_J_relatic

The determinant of $\boldsymbol{G}$ will be denoted by $G=\operatorname{det} \boldsymbol{G}$. The determinant of the transformation then reads $J_{F}=\sqrt{G}$. We use the inverse metric tensor $G^{-1}$ whose entries are denoted by $G^{i j}$. We also introduce for later use the second-kind Christoffel symbols:

$$
\Gamma_{j k}^{i}=\boldsymbol{g}^{i} \cdot \frac{\partial \boldsymbol{g}_{j}}{\partial x^{k}}
$$

with $\boldsymbol{g}^{i}$ the contravariant vector basis. We recall two important properties of the Christoffel symbols:

$$
\frac{\partial G^{i j}}{\partial x^{j}}+G^{i j} \Gamma_{m j}^{m}+\Gamma_{j m}^{i} G^{m j}=0 \text { (Ricci's Lemma), and } \sqrt{G} \Gamma_{i j}^{i}=\frac{\partial \sqrt{G}}{\partial x^{j}}
$$

for the demonstration, see $\frac{\text { Nou }}{41}$. 14

Even though the above relations are valid on general 2D manifolds, all the numerical results in this paper are obtained for a latitude longitude (lat-lon or $\lambda-\varphi$ ) parametrization of the sphere for which the metric tensor and Christoffel symbols of second kind write respectively as:

$$
\boldsymbol{G}=\left[\begin{array}{cc}
R^{2} \cos ^{2} \lambda & 0 \\
0 & R^{2}
\end{array}\right], \boldsymbol{\Gamma}^{1}=\boldsymbol{g}^{1} \cdot \frac{\partial \boldsymbol{g}_{i}}{\partial z^{j}}=\left[\begin{array}{cc}
0 & -\tan \lambda \\
-\tan \lambda & 0
\end{array}\right], \boldsymbol{\Gamma}^{2}=\boldsymbol{g}^{2} \cdot \frac{\partial \boldsymbol{g}_{i}}{\partial z^{j}}=\left[\begin{array}{cc}
\sin \lambda \cos \lambda & 0 \\
0 & 0
\end{array}\right]
$$

with $R$ the sphere radius. 


\section{Appendix A.2. ALE transformation}

In section 3 we have considered an ALE transformation in the parametric domain from the actual curvilinear coordinates $\boldsymbol{x}=\left\{x^{1}, x^{2}\right\}$ with covariant basis $\left\{\boldsymbol{g}_{1}, \boldsymbol{g}_{2}\right\}$ to a set of reference/ALE curvilinear coordinates $\chi=\left\{\chi^{1}, \chi^{2}\right\}$ with covariant basis $\left\{\gamma_{1}, \gamma_{2}\right\}$ :

$$
\boldsymbol{x}=A(\chi, t)
$$

Two different time derivatives have been introduced: the Eulerian time derivative $\frac{\partial}{\partial t}$ and the ALE one $\left.\frac{\partial}{\partial t}\right|_{\chi}$. Then, the spacetime Jacobian matrix and its inverse for the ALE transformation are

$$
\boldsymbol{J}_{A}=\left[\begin{array}{cc}
1 & \frac{\partial t}{\partial \chi^{j}} \\
\left.\frac{\partial x^{i}}{\partial t}\right|_{\chi} & \frac{\partial x^{i}}{\partial \chi^{j}}
\end{array}\right]=\left[\begin{array}{cc}
1 & 0 \\
\sigma^{i} & \frac{\partial x^{i}}{\partial \chi^{j}}
\end{array}\right], \quad \boldsymbol{J}_{A}^{-1}=\left[\begin{array}{cc}
1 & \frac{\partial t}{\partial x^{j}} \\
\frac{\partial \chi^{i}}{\partial t} & \frac{\partial \chi^{i}}{\partial x^{j}}
\end{array}\right]=\left[\begin{array}{cc}
1 & 0 \\
-\Sigma^{i} & \frac{\partial \chi^{i}}{\partial x^{j}}
\end{array}\right]
$$

In the above expression we have used the definitions of covariant mesh velocity, in actual covariant components $\sigma^{i}$ and in reference ones $\Sigma^{i}$. The relationships between the two is given by $\Sigma^{i}=\frac{\partial \chi^{i}}{\partial x^{j}} \sigma^{j}$. One can show that the determinant of the ALE Jacobian reduces to the determinant of the spatial Jacobian $J_{A}=\operatorname{det}\left(\frac{\partial \chi^{i}}{\partial x^{j}}\right)$.

For the time-dependent ALE transformation, Christoffel symbols change with time. We can express

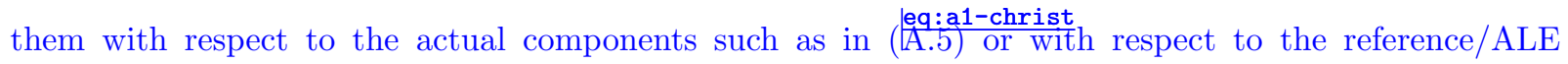
components:

$$
\bar{\Gamma}_{j k}^{i}=\boldsymbol{g}^{i} \cdot \frac{\partial \boldsymbol{g}_{j}(A(\chi, t))}{\partial \chi^{k}}
$$

the relationship between the two being

$$
\begin{aligned}
\Gamma_{j m}^{i} & =\boldsymbol{g}^{i} \cdot \frac{\partial \boldsymbol{g}_{j}}{\partial x^{m}}=\boldsymbol{g}^{i} \cdot \frac{\partial \boldsymbol{g}_{j}(A(\chi, t))}{\partial \chi^{k}} \frac{\partial \chi^{k}}{\partial x^{m}} \\
& =\bar{\Gamma}_{j k}^{i} \frac{\partial \chi^{k}}{\partial x^{m}}
\end{aligned}
$$

Moreover Christoffel symbols relative to the time derivative (denoted by the index 0) can be expressed by chain rule

$$
\bar{\Gamma}_{j 0}^{i}=\left.\boldsymbol{g}^{i} \cdot \frac{\partial \boldsymbol{g}_{j}(A(\boldsymbol{\chi}, t))}{\partial t}\right|_{\chi}=\left.\boldsymbol{g}^{i} \cdot \frac{\partial \boldsymbol{g}_{j}}{\partial x^{m}} \frac{\partial x^{m}}{\partial t}\right|_{\chi}=\Gamma_{j m}^{i} \sigma^{m}=\bar{\Gamma}_{j k}^{i} \frac{\partial \chi^{k}}{\partial x^{m}} \sigma^{m}=\bar{\Gamma}_{j k}^{i} \Sigma^{k}
$$

${ }_{506}$ Finally we distinguish tensor quantities in actual covariant components $\boldsymbol{T}=T^{i j} \boldsymbol{g}_{i} \boldsymbol{g}_{j}$ from tensor quantities in reference covariant components $\boldsymbol{T}=\bar{T}^{i j} \gamma_{i} \gamma_{j}$. 


\section{Appendix B. ALE-SWEs on a 2D manifold derived with a spacetime approach}

With the definitions of Appendix A , the derivation of the ALE-SWEs in curvilinear coordinates is immediate. We consider only momentum conservation since the mass equation is a particular case. Momentum conservation is expressed in terms of the 4-divergence of a second order tensor $\boldsymbol{T}$. We denote, as it is custom, the first row of the tensor as the momentum vector which represents the conserved quantity, e.g. $T^{i 0}=h u^{i}$. Then the divergence in curvilinear reference/ALE coordinates writes

$$
\nabla \cdot \boldsymbol{T}=\left.\frac{1}{J} \frac{\partial}{\partial t}\right|_{\chi}\left(J \overline{h u}^{i}\right)+\frac{1}{J} \frac{\partial}{\partial \chi^{j}}\left(J \bar{T}^{i j}\right)+\bar{\Gamma}_{j 0}^{i} \overline{h u}^{j}+\bar{\Gamma}_{j k}^{i} \bar{T}^{j k}
$$

Note that the total Jacobian $J$ comes from the composition of the spherical and of the ALE transformations, $\boldsymbol{J}=\boldsymbol{J}_{A} \boldsymbol{J}_{F}$ with determinant $J=J_{A} \sqrt{G}$. Moreover we have separated the Christoffel symbols related to time and space variation of the basis vectors.

The last step consists in transforming each $\left\{\overline{h u}^{i}, \bar{T}^{i 1}, \bar{T}^{i 2}\right\}$ into actual components $\left\{h u^{i}, T^{i 1}, T^{i 2}\right\}$

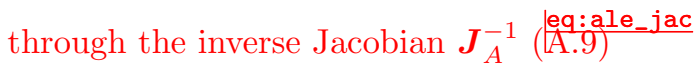

$$
\begin{aligned}
\overline{h u}^{i} & =h u^{i} \\
\bar{T}^{i j} & =\frac{\partial \chi^{j}}{\partial x^{k}} T^{i k}-\Sigma^{j} h u^{i}
\end{aligned}
$$

If we replace the transformed variables (B.3) into B.1] we note that ALE terms related to Christoffel symbols cancel out. This leads to the ALE-SWEs in the reference parametric coordinates:

$$
\left.\frac{1}{J_{A} \sqrt{G}} \frac{\partial}{\partial t}\right|_{\chi}\left(J_{A} \sqrt{G} h u^{i}\right)+\frac{1}{J_{A} \sqrt{G}} \frac{\partial}{\partial \chi^{j}}\left(J_{A} \sqrt{G} \frac{\partial \chi^{j}}{\partial x^{k}} T^{i k}-J_{A} \sqrt{G} \Sigma^{j} h u^{i}\right)+\bar{\Gamma}_{j k}^{i} \frac{\partial \chi^{k}}{\partial x^{m}} T^{j m}=0
$$

which appear in the same form of $\frac{\mathbb{P S}: 03}{18}$ except that momentum components are covariant and not physical.

To get the counterpart of $\begin{gathered}\text { B. } \\ \text { i LE-SWEs-ref }\end{gathered}$ into actual components it suffices to apply chain rule to each term. The advective term then reads

$$
\begin{aligned}
\frac{\partial}{\partial \chi^{j}}\left(J_{A} \sqrt{G} \frac{\partial \chi^{j}}{\partial x^{k}} T^{i k}\right) & =J_{A} \frac{\partial \chi^{j}}{\partial x^{k}} \frac{\partial}{\partial \chi^{j}}\left(\sqrt{G} T^{i k}\right)+\sqrt{G} T^{i k} \frac{\partial}{\partial \chi^{j}}\left(J_{A} \frac{\partial \chi^{j}}{\partial x^{k}}\right) \\
& =J_{A} \frac{\partial}{\partial x^{k}}\left(\sqrt{G} T^{i k}\right)
\end{aligned}
$$

19 The second term on the right hand side is set to zero thanks to the metric identity. The ALE term goes similarly:

$$
\begin{aligned}
\frac{\partial}{\partial \chi^{j}}\left(J_{A} \sqrt{G} \Sigma^{j} h u^{i}\right) & =J_{A} \frac{\partial \chi^{j}}{\partial x^{k}} \frac{\partial}{\partial \chi^{j}}\left(\sqrt{G} \sigma^{k} h u^{i}\right)+\sqrt{G} \sigma^{k} h u^{i} \frac{\partial}{\partial \chi^{j}}\left(J_{A} \frac{\partial \chi^{j}}{\partial x^{k}}\right) \\
& =J_{A} \frac{\partial}{\partial x^{k}}\left(\sqrt{G} \sigma^{k} h u^{i}\right)
\end{aligned}
$$




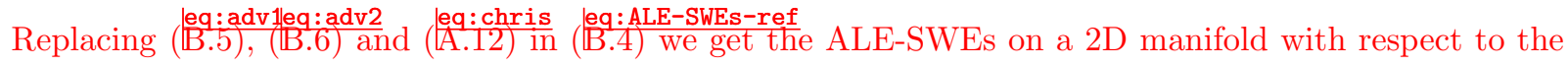
actual parametric coordinates:

$$
\left.\frac{\partial}{\partial t}\right|_{\chi}\left(\sqrt{G} J_{A} h u^{i}\right)+J_{A} \frac{\partial}{\partial x^{j}}\left(\sqrt{G} T^{i j}-\sqrt{G} h u^{i} \sigma^{j}\right)+\sqrt{G} J_{A} \Gamma_{j k}^{i} T^{j k}=0
$$

524

Except for the physical source terms, the above set of equations coincides with 10 mom-as equation discretized in this article. In section $\frac{10 \mathrm{As}}{4}$ it has has been obtained by a more classical procedure combining transport formulas and the GCL.

\section{Appendix C. Proof of proposition 1}

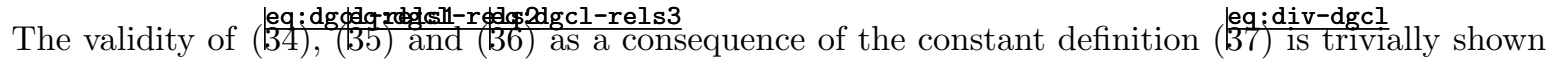
using (3i1) and the definition of the integrated edge velocities (32) - (33). Concerning the accuracy of the projection, we remark that by virtue of the mean value theorem

$$
|\overline{\mathcal{K}}|=\int_{\bar{K}} \sqrt{G}(\overline{\boldsymbol{x}}) d \overline{\boldsymbol{x}}=|\bar{K}| \sqrt{G}\left(\overline{\boldsymbol{x}}_{*}\right) \rightarrow \int_{\bar{K}}\left(\sqrt{G}(\overline{\boldsymbol{x}})-\sqrt{G}\left(\overline{\boldsymbol{x}}_{*}\right)\right) d \overline{\boldsymbol{x}}=0
$$

for some $\overline{\boldsymbol{x}}_{*}$ within the element. Estimating the error of $\frac{1}{38}$ g gal-mass-d is equivalent to estimating the error of

$$
\int_{\bar{K}} \varphi_{i}(\overline{\boldsymbol{x}}) f_{\mathrm{h}}(\overline{\boldsymbol{x}}) \sqrt{G}(\overline{\boldsymbol{x}}) d \overline{\boldsymbol{x}} \approx \int_{\bar{K}} \varphi_{i}(\overline{\boldsymbol{x}}) f_{\mathrm{h}}(\overline{\boldsymbol{x}}) \sqrt{G}\left(\overline{\boldsymbol{x}}_{*}\right) d \overline{\boldsymbol{x}}
$$

To this end, we follow the consistency analysis used e.g. in $\frac{\mid A R: 17 \text {. Dec: } 17}{42}$, we take the difference between the two expressions, sum over all the elements, multiply by the sampled nodal values of an arbitrary test function $\psi \in C^{r}(r \geq 1)$, and sum over all the nodes. We end up with the following global error

$$
\epsilon:=\left|\sum_{K} \sum_{j \in \bar{K}} \int_{\bar{K}} \psi_{j} \varphi_{j}(\overline{\boldsymbol{x}}) f_{\mathrm{h}}(\overline{\boldsymbol{x}})\left(\sqrt{G}(\overline{\boldsymbol{x}})-\sqrt{G}\left(\overline{\boldsymbol{x}}_{*}\right)\right) d \overline{\boldsymbol{x}}\right|=\left|\sum_{K} \int_{\bar{K}} \psi_{\mathrm{h}}(\overline{\boldsymbol{x}}) f_{\mathrm{h}}(\overline{\boldsymbol{x}})\left(\sqrt{G}(\overline{\boldsymbol{x}})-\sqrt{G}\left(\overline{\boldsymbol{x}}_{*}\right)\right) d \overline{\boldsymbol{x}}\right|
$$

The trick is now to use $\frac{\text { a }}{\text { r. }}$ to to remove nodal values $\psi_{j} f_{j}$, leading to

$$
\epsilon=\left|\sum_{K} \sum_{j \in K} \int_{\bar{K}} \frac{\psi_{\mathrm{h}}(\overline{\boldsymbol{x}}) f_{\mathrm{h}}(\overline{\boldsymbol{x}})-\psi_{j} f_{j}}{3}\left(\sqrt{G}(\overline{\boldsymbol{x}})-\sqrt{G}\left(\overline{\boldsymbol{x}}_{*}\right)\right) d \overline{\boldsymbol{x}}\right|
$$

We now use the relation $(a b)_{2}-(a b)_{1}=\left(a_{2}+a_{1}\right)\left(b_{2}-b_{1}\right) / 2+\left(a_{2}-a_{1}\right)\left(b_{2}+b_{1}\right) / 2$ and recast the error as

$$
\epsilon=\left|\sum_{K} \sum_{j \in K} \int_{\bar{K}}\left(\frac{\psi_{\mathrm{h}}+\psi_{j}}{6}\left(f_{\mathrm{h}}(\overline{\boldsymbol{x}})-f_{j}\right)+\left(\psi_{\mathrm{h}}-\psi_{j}\right) \frac{f_{\mathrm{h}}(\overline{\boldsymbol{x}})+f_{j}}{6}\right)\left(\sqrt{G}(\overline{\boldsymbol{x}})-\sqrt{G}\left(\overline{\boldsymbol{x}}_{*}\right)\right) d \overline{\boldsymbol{x}}\right|
$$

We can now use standard arguments to bound this quantity as

$$
\begin{aligned}
\epsilon & \leq \sum_{K} \sum_{j \in K} \int_{\bar{K}}\left(\frac{\left|\psi_{\mathrm{h}}+\psi_{j}\right|}{6}\left|f_{\mathrm{h}}(\overline{\boldsymbol{x}})-f_{j}\right|+\left|\psi_{\mathrm{h}}-\psi_{j}\right| \frac{\left|f_{\mathrm{h}}(\overline{\boldsymbol{x}})+f_{j}\right|}{6}\right)\left|\sqrt{G}(\overline{\boldsymbol{x}})-\sqrt{G}\left(\overline{\boldsymbol{x}}_{*}\right)\right| d \overline{\boldsymbol{x}} \\
& \leq c_{1}\left|S^{2}\right|\left(\|\psi\|_{L^{\infty}} \sup _{j}\left\|\partial_{x^{j}} f\right\|_{L^{\infty}}+\sup _{j}\left\|\partial_{x^{j}} \psi\right\|_{L^{\infty}}\|f\|_{L^{\infty}}\right) \sup _{j}\left\|\partial_{x^{j}} \sqrt{G}\right\|_{L^{\infty}} \mathrm{h}^{2}
\end{aligned}
$$




\section{Appendix D. Stabilized FEM and RD in moving curvilinear coordinates}

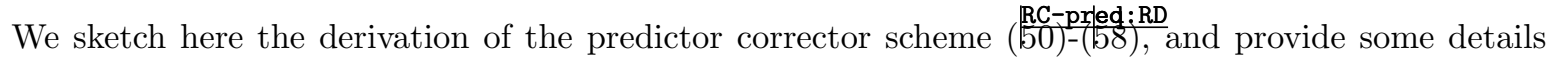
concerning the computation of the mass matrices with and without the simplified quadrature of proposition 1.

We start with the following explicit unstabilized predictor:

$$
\int_{S^{2}} \varphi_{i} \sqrt{G} J_{A}^{n+1} \mathrm{w}^{*} d \chi-\int_{S^{2}} \varphi_{i} \sqrt{G} J_{A}^{n} \mathrm{w}^{n} d \chi+\Delta t \int_{S^{2}} \varphi_{i} \bar{J}_{A}\left\{\partial_{x^{j}}\left(\mathrm{~F}^{j}-\sqrt{G} \sigma^{j} \mathrm{w}\right)^{n}+\sqrt{G} \mathcal{S}^{n}\right\} d \chi=0
$$

which we recast as

$$
\begin{aligned}
\int_{S^{2}} \varphi_{i} \sqrt{G} J_{A}^{n+1}\left(\mathrm{w}^{*}-\mathrm{w}^{n}\right) d \boldsymbol{\chi} & +\Delta t \int_{S^{2}} \varphi_{i} \sqrt{G} \frac{J_{A}^{n+1}-J_{A}^{n}}{\Delta t} \mathrm{w}^{n} d \chi \\
& +\Delta t \int_{S^{2}} \varphi_{i} \bar{J}_{A}\left\{\partial_{x^{j}}\left(\mathrm{~F}^{j}-\sqrt{G} \sigma^{j} \mathrm{w}\right)^{n}+\sqrt{G} \mathcal{S}^{n}\right\} d \boldsymbol{\chi}=0 .
\end{aligned}
$$

For a fixed mesh, $J_{A}=1$ and $\sigma^{j}=0$. For a moving mesh (and only for a moving mesh) we assume that the mass matrices are computed following proposition 1. Otherwise, on fixed meshes we can either

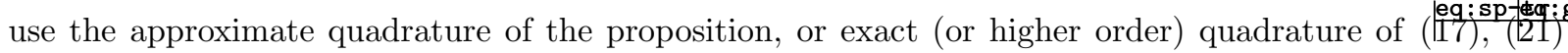

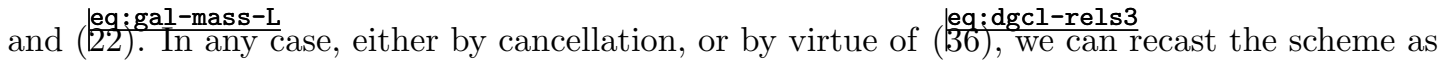

$$
\int_{S^{2}} \varphi_{i} \sqrt{G} J_{A}^{n+1}\left(\mathrm{w}^{*}-\mathrm{w}^{n}\right) d \chi+\Delta t \int_{S^{2}} \varphi_{i} \bar{J}_{A}\left\{\partial_{x^{j}}\left(\mathrm{~F}^{j}\right)^{n}-\sqrt{G} \sigma^{j} \partial_{x^{j}} \mathrm{w}^{n}+\sqrt{G} \mathcal{S}^{n}\right\} d \chi=0
$$

As in $\frac{\operatorname{ArR}: 14}{33}$ we 1) stabilize the predictor step using an incomplete residual which does not involve the time increment, and 2) lump the Galerkin mass matrix to obtain:

$$
\begin{aligned}
\left(\sum_{K \ni i} \mathcal{K}_{i}\right)\left(\mathrm{w}_{i}^{*}-\mathrm{w}_{i}^{n}\right) & +\Delta t \int_{S^{2}} \varphi_{i} \bar{J}_{A}\left\{\partial_{x^{j}}\left(\mathrm{~F}^{j}\right)^{n}-\sqrt{G} \sigma^{j} \partial_{x^{j}} \mathrm{w}^{n}+\sqrt{G} \mathcal{S}^{n}\right\} d \boldsymbol{\chi} \\
& +\Delta t \sum_{K \ni i} \int_{K_{0}} \gamma_{i} \bar{J}_{A}\left\{\partial_{x^{j}}\left(\mathrm{~F}^{j}\right)^{n}-\sqrt{G} \sigma^{j} \partial_{x^{j}} \mathrm{w}^{n}+\sqrt{G} \mathcal{S}^{n}\right\} d \chi=0
\end{aligned}
$$

where $\gamma_{i}$ is a properly defined stabilization bubble. In particular, following $\frac{R i A}{46}, 10$, ArR: 14 it is assumed that for $\mathrm{RD}$ schemes the definition of the right hand side is such that

$$
\int_{K_{0}}\left(\varphi_{i}+\gamma_{i}\right) \bar{J}_{A}\left\{\partial_{x^{j}}\left(\mathrm{~F}^{j}\right)^{n}-\sqrt{G} \sigma^{j} \partial_{x^{j}} \mathrm{w}^{n}+\sqrt{G} \mathcal{S}^{n}\right\} d \boldsymbol{\chi}=\tilde{\Phi}_{i}^{K}
$$




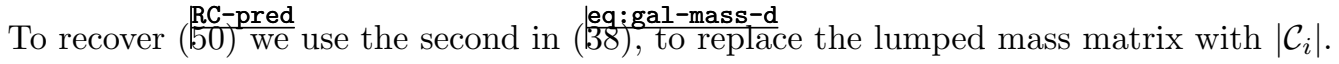

The corrector step is derived in a similar manner. We start from the second stage Runge-Kutta iteration for the unstabilized Galerkin scheme:

$$
\begin{array}{r}
\int_{S^{2}} \varphi_{i} \sqrt{G} J_{A}^{n+1} \mathrm{w}^{n+1} d \chi-\int_{S^{2}} \varphi_{i} \sqrt{G} J_{A}^{n} \mathrm{w}^{n} d \chi \\
+\frac{\Delta t}{2} \int_{S^{2}} \varphi_{i} \bar{J}_{A}\left\{\partial_{x^{j}}\left(\mathrm{~F}^{j}-\sqrt{G} \sigma^{j} \mathrm{w}\right)^{n}+\sqrt{G} \mathcal{S}^{n}\right\} d \chi \\
+\frac{\Delta t}{2} \int_{S^{2}} \varphi_{i} \bar{J}_{A}\left\{\partial_{x^{j}}\left(\mathrm{~F}^{j}-\sqrt{G} \sigma^{j} \mathrm{w}\right)^{*}+\sqrt{G} \mathcal{S}^{*}\right\} d \chi=0
\end{array}
$$

$$
\begin{aligned}
& \int_{S^{2}} \varphi_{i} \sqrt{G} J_{A}^{n+1} \mathrm{w}^{n+1} d \chi-\int_{S^{2}} \varphi_{i} \sqrt{G} J_{A}^{n} \mathrm{w}^{n} d \chi+\frac{\Delta t}{2} \int_{S^{2}} \varphi_{i} \bar{J}_{A}\left\{\partial_{x^{j}}\left(\mathrm{~F}^{j}-\sqrt{G} \sigma^{j} \mathrm{w}\right)^{n}+\sqrt{G} \mathcal{S}^{n}\right\} d \chi \\
&+\frac{\Delta t}{2} \int_{S^{2}} \varphi_{i} \bar{J}_{A}\left\{\partial_{x^{j}}\left(\mathrm{~F}^{j}-\sqrt{G} \sigma^{j} \mathrm{w}\right)^{*}+\sqrt{G} \mathcal{S}^{*}\right\} d \chi \\
&+\sum_{K \ni i} \int_{K_{0}} \gamma_{i}\left\{\sqrt{G} J_{A}^{n+1} \mathrm{w}^{*}-\sqrt{G} J_{A}^{n} \mathrm{w}^{n}+\frac{\Delta t}{2} \bar{J}_{A}\left[\partial_{x^{j}}\left(\mathrm{~F}^{j}-\sqrt{G} \sigma^{j} \mathrm{w}\right)+\sqrt{G} \mathcal{S}\right]^{n}\right. \\
&\left.+\frac{\Delta t}{2} \bar{J}_{A}\left[\partial_{x^{j}}\left(\mathrm{~F}^{j}-\sqrt{G} \sigma^{j} \mathrm{w}\right)+\sqrt{G} \mathcal{S}\right]^{*}\right\} d \chi=0
\end{aligned}
$$

We now add and remove the term $\int_{S^{2}} \varphi_{i} \sqrt{G} J_{A}^{n+1} \mathrm{w}^{*} d \chi$ to obtain the following error correction form 544 $\frac{R i A: 10}{46}$

$$
\begin{aligned}
\int_{S^{2}} \varphi_{i} \sqrt{G} J_{A}^{n+1}\left(\mathrm{w}^{n+1}-\mathrm{w}^{*}\right) d \chi= & -\sum_{K \ni i} \int_{K_{0}}\left(\varphi_{i}+\gamma_{i}\right)\left\{\sqrt{G} J_{A}^{n+1} \mathrm{w}^{*}-\sqrt{G} J_{A}^{n} \mathrm{w}^{n}\right. \\
& +\frac{\Delta t}{2} \bar{J}_{A}\left[\partial_{x^{j}}\left(\mathrm{~F}^{j}-\sqrt{G} \sigma^{j} \mathrm{w}\right)+\sqrt{G} \mathcal{S}\right]^{n} \\
& \left.+\frac{\Delta t}{2} \bar{J}_{A}\left[\partial_{x^{j}}\left(\mathrm{~F}^{j}-\sqrt{G} \sigma^{j} \mathrm{w}\right)+\sqrt{G} \mathcal{S}\right]^{*}\right\} d \chi
\end{aligned}
$$

For piecewise constant (per element) values of $\gamma_{i}$, we can see that in general the form of the full mass matrix on a manifold is

$$
m_{i j}^{K}=\left(m_{i j}^{G A L}\right)^{K}+\mathcal{K}_{j} \gamma_{i}
$$

If proposition 1 is valid, the last expression becomes

$$
m_{i j}^{K}=|\mathcal{K}| \frac{\delta_{i j}+1}{12}+\frac{|\mathcal{K}|}{3} \gamma_{i}
$$

${ }_{548}$ The standard RD mass matrix corrections are obtained by setting $\gamma_{i}=\boldsymbol{\beta}_{i}^{K}-1 / 3$, while the final form (18C-corr of the scheme is obtained by recalling the hypothesis $\left[\frac{R i A}{46}\right]:$ : 


$$
\begin{aligned}
\Phi_{i}^{K}=\int_{K_{0}}\left(\varphi_{i}+\gamma_{i}\right)\left\{\frac{\sqrt{G} J_{A}^{n+1} \mathrm{w}^{*}-\sqrt{G} J_{A}^{n} \mathrm{w}^{n}}{\Delta t}+\frac{1}{2} \bar{J}_{A}\left[\partial_{x^{j}}\left(\mathrm{~F}^{j}-\sqrt{G} \sigma^{j} \mathrm{w}\right)+\sqrt{G} \mathcal{S}\right]^{n}\right. \\
\left.+\frac{1}{2} \bar{J}_{A}\left[\partial_{x^{j}}\left(\mathrm{~F}^{j}-\sqrt{G} \sigma^{j} \mathrm{w}\right)+\sqrt{G} \mathcal{S}\right]^{*}\right\} d \chi
\end{aligned}
$$

550

551

ArR: 175

553

Ros : 045

555

Ros : 065

557

UIJ : 195\$

559

Cas : 186

561

Comb : 096

563

Bern:0964

565

566

Ros : 136

568

569

RiB : 09

571

r117

573

\section{References}

[1] L. Arpaia, M. Ricchiuto, r-adaptation for shallow water flows: conservation, well balancedness, efficiency, Computers \& Fluids 160 (2018) 175-203.

[2] J. A. Rossmanith, D. S. Bale, R. J. LeVeque, A wave propagation algorithm for hyperbolic systems on curved manifold, J. Comput. Phys. 199 (2004) 631-662.

[3] J. A. Rossmanith, A wave propagation algorithm for hyperbolic systems the sphere, J. Comput. Phys. 213 (2006) 629-658.

[4] P. A. Ullrich, C. Jablonowksi, B. Van-Leer, High-order finite-volume methods for the shallow water equations on the sphere, J. Comput. Phys. 229 (2010) 6104-6134.

[5] M. Castro, S. Ortega, C. Parés, Well-balanced methods for the shallow water equations in spherical coordinates, Computers \& Fluids 169 (2018) 129-140.

[6] R. Comblen, S. Legrand, E. Deleersnijder, V. Legat, A finite element method for solving the shallow water equations on the sphere, Ocean Modelling 28 (2009) 12-23.

[7] P. E. Bernard, J. F. Remacle, R. Comblen, V. Legat, K. Hillewaert, High-order discontinous Galerkin schemes on general 2D manifolds applied to the shallow water eqautions, J. Comput. Phys. 228 (2009) $6514-6535$.

[8] J. A. Rossmanith, Residual distribution schemes for hyperbolic balance laws in generalized coordinates, in: Numerical Modeling of Space Plasma Flows, Vol. 359 of ASP Conference Series, Nikolai V. Pogorelov and Gary P. Zank, 2013.

[9] M. Ricchiuto, A. Bollerman, Stabilized residual distribution for shallow water simulations, J. Comput. Phys 228 (2009) 1071-111.

[10] M. Ricchiuto, On the C-property and generalized C-property of residual distribution for the shallow water equations, J. Sci. Comput. 48 (2011) 304-318. 
Ric : 157

575

SkK : 927

577

McU : 157

579

580

Fer:168

582

583

LGB : 1 1 8

585

Pop: 118

[11] M. Ricchiuto, An explicit residual based approach for shallow water flows, J. Comput. Phys. 80 (2015) 306-344.

[12] W. Skamarock, J. Klemp, Adaptive grid refinement for two-dimensional and three-dimensional nonhydrostatic atmospheric flow, Mon. Weather Rev. 121 (1992) 788-804.

[13] P. McCorquodale, P. Ullrich, H. Johansen, P. Colella, An adaptive multi-block high-order finitevolume method for soliving the shallow water equations on the sphere, Comm. App. Math. and Comp. Sci. 10 (2) (2015) 121-162.

[14] J. Ferguson, C. Jablonowski, H. Johansen, P. McCorquodale, P. Colellaand, P. Ullrich, Analyzing the adaptive mesh refinement (AMR) characteristics of a high-order 2D cubed-sphere shallow-water model, Mon. Weather Rev. 144 (12) (2016) 4641-4666.

[15] R. LeVeque, D. George, M. Berger, Tsunami modelling with adaptively refined finite volume methods, Acta Numerica 20 (2011) 211-289.

[16] S. Popinet, Quadtree-adaptive tsunami modelling, Ocean Dynamics 61 (9) (2011) 1261-1285.

[17] C. W. Hirt, A. A. Amsden, J. L. Cook, An Arbitrary Lagrangian-Eulerian computing method for all flow speeds, J. Comput. Phys. 14 (3) (1974) 227-253.

[18] J. Prusa, P. Smolarkiewicz, An all-scale anelastic model for geophysical flows: dynamic grid derformation, J. Comp. Phys. 190 (2003) 601-622.

[19] C. Kuhnlein, P. Smolarkiewicz, A. Dornbrack, Modelling atmospheric flows with adaptive moving meshes, J. Comp. Phys. 231 (2012) 2741-2763.

[20] F. Zhou, G. Chen, Y. Huang, J. Z. Yang, H. Feng, An adaptive moving finite volume scheme for modeling flood inundation over dry and complex topography, Water Resour. Res. 49 (2013) 19141928.

[21] Y. Bazilevs, V. M. Calo, T. J. R. Hughes, Y. Zhang, Isogeometric fluid-structure interaction: theory, algorithms, and computations, Comput. Mech. 43 (2008) 3-37.

[22] Y. Bazilevs, K. Takizawa, T. F. Tezduyar, Computational Fluid-Structure Interaction. Methods and applications, Wiley, 2012.

[23] V. A. Dobrev, T. V. Kolev, R. N. Rieben, High-order curvilinear finite element methods for Lagrangian hydrodynamics, SIAM J. Sci. Comput. 34 (2012) B606-B641. 
[24] W. Boscheri, M. Dumbser, High order accurate direct Arbitrary-Lagrangian-Eulerian ADER-WENO finite volume schemes on moving curvilinear unstructured meshes, Computers \& Fluids 136 (2016) $48-66$.

[25] W. Boscheri, M. Dumbser, A direct Arbitrary Lagrangian Eulerian ADER-WENO finite volume scheme on unstructured tetrahedral meshes for conservative and non-conservative hyperbolic systems in 3D, J. Comput. Phys. 275 (2014) $484-523$.

[26] F. Vilar, P.-H. Maire, R. Abgrall, A discontinuous Galerkin discretization for solving the twodimensional gas dynamics equations written under total Lagrangian formulation on general unstructured grids, J. Comput. Phys. 276 (2014) $188-234$.

[27] S. A. Teukolsky, Formulation of discontinuous Galerkin methods for relativistic astrophysics, J. Comput. Phys. 312 (2016) 333-356.

[28] P. Thomas, C. Lombard, Geometric conservation law and its application to flow computations on moving grids, AIAA Journal (1979) 1030-1037.

[29] M. Lesoinne, C. Farhat, Geometric conservation laws for flow problems with moving boundaries and deformable meshes, and their impact on aeroelastic computations, Comput. Meth. Appl. Mech. Engrg 134 (1996) 71-90.

[30] H. Guillard, C. Farhat, On the significance of the geometric conservation law for flow computations on moving meshes, Comput. Meth. Appl. Mech. Engrg 190 (2000) 1467-1482.

[31] S. Etienne, A. Garon, D. Pelletier, Geometric conservation law and finite element methods for 3D unsteady simulations of incompressible flow, in: 39th AIAA Fluid Dynamics Conference, 22-25 June 2009. San Antonio, Texas, 2009.

[32] S. Savidis, D. Aubram, F. Rackwit, Arbitrary Lagrangian Eulerian finite element formulation for geotechnical construction processes, Journal of Theoretical and Applied Mechanics 38 (2008) 165174.

[33] L. Arpaia, M. Ricchiuto, R. Abgrall, An ALE formulation for explicit Runge-Kutta residual distribution, J. Sci. Comput. 190 (34) (2014) 1467-1482.

[34] R. LeVeque, Wave propagation algorithm for multidimensional hyperbolic systems, J. Comput. Phys. 131 (1997) 3279-353. 
Tru : 66зф

631

Wil:073

633

KaS : 0434

635

RoI : 96зф

637

BeV : 943\$

639

ZhC : 134

641

Ngu : 144

643

MaY : 0644

645

shoelacen

Dec: 174

648

AR: 174

650

RiA : 165

652

hr11 55

654

$\operatorname{shr} 135$

656

[35] C. Truesdell, The mechanical foundations of elasticity and fluid dynamics, Gordon and Breach Science Publicher Inc., 1966.

[36] D. Williamson, The evolution of dynamical cores for global atmospheric models, J. Meteorol. Soc. Jpn. 85 (2007) 241-269.

[37] A. Kageyama, T. Sato, "Yin-Yang grid": An overset grid in spherical geometry, Geochem. Geophys. Geosyst. 5 (2004) Q09005.

[38] C. Ronchi, R. Iacono, P. S. Paolucci, The "cubed sphere": A new method for the solution of partial differential equations in spherical geometry, J. Comput. Phys. 124 (1996) 93-114.

[39] A. Bermudez, M. E. Vazquez-Cendon, Upwind methods for hyperbolic conservation laws with source terms, Comput. Fluids 235 (8) (1994) 1049-1071.

[40] F. Zhou, G. Chen, S. Noelle, H. Guo, A well-balanced stable GRP scheme for shallow water equations for adaptive unstructured triangular meshes, Int. J. Numer. Meth. Fl. 73 (2013) 266-283.

[41] H. Nguyen-Shafer, J.-P. Schmidth, Tensor Analysis and Elementary Differential Geometry for Physicist and Engineers, Vol. 21 of Mathematical Engineering, Springer, 2014.

[42] D. J. Mavriplis, Z. Yang, Construction of the discrete geometric conservation law for high-order time-accurate simulations on dynamic meshes, J. Comput. Phys. 213 (2006) 557-573.

[43] B. Braden, The surveyor's area formula, The College Mathematics Journal 17 (4) (1986) 326-337.

[44] H. Deconinck, M. Ricchiuto, Residual distribution schemes: Foundations and analysis, in: J. Wiley, Sons (Eds.), Encyclopedia of Computational Mechanics, second edition, 2017.

[45] R. Abgrall, M. Ricchiuto, High order methods for CFD, in: R. d. B. Erwin Stein, T. J. Hughes (Eds.), Encyclopedia of Computational Mechanics, Second Edition, John Wiley and Sons, 2017.

[46] M. Ricchiuto, R. Abgrall, Explicit Runge-Kutta residual distribution schemes for time dependent problems: Second order case, J. Comput. Phys. 229 (16) (2010) 5653-5691.

[47] M. Hubbard, M. Ricchiuto, Discontinuous upwind residual distribution: A route to unconditional positivity and high order accuracy, Computers \& Fluids 46 (1) (2011) 263 - 269.

[48] D. Sármány, M. Hubbard, M. Ricchiuto, Unconditionally stable space-time discontinuous residual distribution for shallow-water flows, Journal of Computational Physics 253 (2013) 86 - 113. 
hrs265

[49] M. Hubbard, M. Ricchiuto, D. Sármány, Space-time residual distribution on moving meshes, Computers \& Mathematics with Applicationsdoi:https://doi.org/10.1016/j.camwa.2019.09.019

[50] R. Abgrall, Essentially non-oscillatory residual distribution schemes for hyperbolic problems, J. Comput. Phys. 214 (2006) 773-808.

[51] C. de Boor, Good Approximations by Splines with Variable Knots II, Vol. 363, Springer, Berlin, 1973.

[52] H. Ceniceros, T. Hou, An efficient dynamically adaptive mesh for potentially singular solutions, J. Comput. Phys. 172 (2001) 609-639.

[53] H. Tang, T. Tang, Adaptive mesh methods for one and two-dimensional hyperbolic conservation laws, SIAM J. Numer. Anal. 41 (2) (2003) 487-515.

[54] C. Jin, K. Xu, An adaptive grid method for two-dimensional viscous flows, J. Comput. Phys. 218 (1) (2006) $68-81$.

[55] G. Chen, H. Tang, P. Zhang, Second-order accurate Godunov scheme for multicomponent flows on moving triangular meshes, J. Sci. Comput. 34 (2008) 64-86.

[56] G. Ni, S. Jiang, K. Xu, Remapping-free ALE-type kinetic method for flow computations, J. Comp. Phys. 228 (2009) 3154-3171.

[57] L. Pan, K. Xu, Generalized coordinate transformation and gas-kinetic scheme, J. Comput. Phys. 287 (2015) $207-225$.

[58] H. Weller, P. Browne, C. Budd, M. Cullen, Mesh adaptation on the sphere using optimal transport and the numerical solution of a Monge-Ampere type equation, J. Comput. Phys. 308 (2016) 102-123.

[59] A. McRae, C. Cotter, C. Budd, Optimal-transport-based mesh adaptivity on the plane and sphere using finite elements, SIAM J. Sci. Comp. 40 (2) (2018) A1121-A1148.

[60] Y. Di, R. Li, T. Tang, P. Zhang, Moving mesh methods for singular problems on a sphere using perturbed harmonic mappings, J. Sci. Comput. 28 (4) (2006) 1490-1508.

[61] W. Huang, R. D. Russell, Analysis of moving mesh partial differential equations with spatial smoothing, J. Numer. Anal. 34 (3) (1997) 1106-1126.

[62] C. J. Budd, W. Huang, R. D. Russell, Adaptivity with moving grids, Cambridge University Press, 2009, Ch. 1, pp. 1-131. 
[63] A. M. Winslow, Numerical solution of the quasi-linear Poisson equation, J. Comput. Phys. 1 (1967) $149-172$.

[64] C. Geuzaine, J.-F. Remacle, Gmsh: a three-dimensional finite element mesh generator with built-in pre- and post-processing facilities, http://www.geuz.org/gmsh (1997-2017).

[65] D. L. Williamson, J. B. Drake, J. J. Hack, R. Jakob, P. N. Swarztrauber, A standard test set for numerical approximations to the shallow water equations in spherical geometry, J. Comput. Phys. 102 (1992) 211-224.

[66] J. Galewsky, R. Scott, L. Polvani, An initial-value problem for testing numerical models of the global shallow-water equations, Tellus A: Dynamic Meteorology and Oceanography (56:5) (2004) 429-440.

[67] S. LeRoy, A. Lemoine, R. Pedreros, M. Rousseau, Tohoku-Oki 2011 tsunami high-resolution modeling and sensitivity to the rupture complexity: Kamaishi and Sendai areas, in: TANDEM Workshop on French Japanese week on disaster risk reduction, Oct 2017, Tokyo, Japan, 2017.

[68] K. Satake, Y. Fujii, T. Harada, Y. Namegaya, Time and space distribution of coseismic slip of the 2011 tohoku earthquake as inferred from tsunami waveform data, Bull. Seismol. Soc. Am. 103 (2013) 1473-1492.

[69] G. Gayer, S. Leschka, I. Nohren, O. Larsen, H. Gunther, Unconditionally stable spacetime discontinuous residual distribution for shallow-water flows, Nat. Hazards Earth Syst. Sci. 10 (2010) 1679-1687.

[70] C. Chen, Z. Lai, R. Beardsley, J. Sasaki, J. Lin, H. Lin, R. Ji, Y. Sun, The March 11, 2011 tohoku M9.0 earthquake-induced tsunami and coastal inundation along the Japanese coast: A model assessment, Prog. Oceanogr. 123 (2014) 84-104.

[71] F. Loevholt, G. Kaiser, S. Glimsdal, L. Scheele, C. Harbitz, , G. Pedersen, Modeling propagation and inundation of the 11 March 2011 Tohoku tsunami, Nat. Hazards Earth Syst. Sci. 12 (2012) 1017-1028.

[72] T. Shimozono, S. Sato, A. Okayasu, Y. Tajima, H. Fritz, , H. Liu, T. Takagawa, Propagation and inundation characteristics of the 2011 Tohoku tsunami on the central Sanriku coast, Coast. Eng. J. 54 (2012) 1017-1028. 